\title{
Biomaterials for Sequestration of Growth Factors and Modulation of Cell Behavior
}

\author{
Simão P. B. Teixeira, Rui M. A. Domingues, * Mariya Shevchuk, Manuela E. Gomes, * \\ Nicholas A. Peppas, * and Rui L. Reis
}

Growth factors (GFs) are proteins secreted by cells that regulate a variety of biological processes. Although they have long been proposed as potent therapeutic agents, their administration in a soluble form has proven costly and ineffective due to their short half-lives in biological environments. Biomaterial-based approaches are increasingly sought as alternatives to improve the efficacy or, ideally, replace the need for exogenous administration of GFs in regenerative medicine strategies. The means by which these systems evolve from biomaterials for conventional controlled release of GFs to the recent extracellular matrix (ECM)-inspired approaches for sequestering these labile molecules and regulating their spatiotemporal activity and presentation are reviewed. Focus is placed on biomaterials functionalized either with ECM components, which show promiscuous GF binding, or with targeted GF ligands (antibodies, aptamers, or peptides). The potential of synthetic platforms with abiotic affinity as cost-effective alternatives to the current biological ligands is also discussed. Overall, the various GF sequestering systems developed so far have remarkably improved the activity of GFs at reduced doses and, in some cases, completely avoided the need for their exogenous administration to guide cell fates. These bioinspired concepts thus enable the rational exploration of the full therapeutic potential of GFs in regenerative medicine.

\section{Introduction}

Growth factors (GFs) are soluble proteins secreted by cells that act in an autocrine and paracrine manner by binding to specific receptors at the cellular surface. They regulate a set of behaviors, from survival to migration, proliferation, and differentiation, ${ }^{[1]}$ playing a crucial role in tissue development ${ }^{[2]}$ and healing. ${ }^{[3,4]}$ Thus, since their discovery, GFs have been identified as potent therapeutic agents, in particular, for regenerative medicine and tissue engineering applications.[5] This has led to widespread research on the topic, and to their use in preclinical and clinical trials, with over 1600 entries registered on ClinicalTrials.gov for the use of GFs as part of interventions. ${ }^{6]}$

However, regulatory approval and commercialization have lagged far behind. The few GFs that have been clinically approved by the U.S. Food and Drug Administration (FDA) are bone morphogenic proteins
S. P. B. Teixeira, Dr. R. M. A. Domingues, Prof. M. E. Gomes, Prof. R. L. Reis 3B's Research Group

I3Bs-Research Institute on Biomaterials

Biodegradables and Biomimetics

University of Minho

Headquarters of the European Institute of Excellence

on Tissue Engineering and Regenerative Medicine

AvePark, Parque de Ciência e Tecnologia

Zona Industrial da Gandra

Barco, Guimarães, 4805-017 Portugal

E-mail: rui.domingues@i3bs.uminho.pt; megomes@i3bs.uminho.pt

S. P. B. Teixeira, Dr. R. M. A. Domingues, Prof. M. E. Gomes, Prof. R. L. Reis ICVS/3B's-PT Government Associate Laboratory

Braga, Guimarães, Portugal

S. P. B. Teixeira, Dr. R. M. A. Domingues, Prof. M. E. Gomes, Prof. R. L. Reis The Discoveries Centre for Regenerative and Precision Medicine

Headquarters at University of Minho, AvePark

Barco, Guimarães, 4805-017 Portugal

M. Shevchuk, Prof. N. A. Peppas

McKetta Department of Chemical Engineering

The University of Texas at Austin

Austin, TX 78712-1801, USA

E-mail: peppas@che.utexas.edu

The ORCID identification number(s) for the author(s) of this article can be found under https://doi.org/10.1002/adfm.201909011.
M. Shevchuk, Prof. N. A. Peppas

Institute for Biomaterials

Drug Delivery, and Regenerative Medicine

The University of Texas at Austin

Austin, TX 78712-1801, USA

M. Shevchuk, Prof. N. A. Peppas

Department of Biomedical Engineering

The University of Texas at Austin

Austin, TX 78712-1801, USA

M. Shevchuk, Prof. N. A. Peppas

Department of Pediatrics

Dell Medical School

The University of Texas at Austin

Austin, TX 78712-1801, USA

M. Shevchuk, Prof. N. A. Peppas

Department of Surgery and Perioperative Care

Dell Medical School

The University of Texas at Austin

Austin, TX 78712-1801, USA

M. Shevchuk, Prof. N. A. Peppas

Division of Molecular Pharmaceutics and Drug Delivery

College of Pharmacy

The University of Texas at Austin

Austin, TX 78712-1801, USA

DOI: 10.1002/adfm.201909011 
2 (BMP-2) and 7 (BMP-7) for lumbar spine fusion and bone fracture; platelet-derived growth factor-BB (PDGF-BB) for the enhancement of granulation tissue formation; and keratinocyte growth factor (KGF) for prevention and treatment of mucositis in cancer patients who receive high doses of chemotherapy and radiation therapy. ${ }^{[5,7]}$ Other approved GF therapeutics include the use of fibroblast growth factor-2 (FGF-2) in Japan and China $^{[8]}$ and epidermal growth factor (EGF) in Latin America and parts of $\mathrm{Asia}^{[9]}$ for the treatment of wounds. This degree of translational failure demonstrates that a number of limitations are impeding the success of GF therapeutics.

Major shortcomings include short half-lives in circulation, susceptibility to deactivation and degradation by enzymes, and rapid rates of internalization. ${ }^{[5,10,11]}$ For example, the halflife of vascular endothelial growth factor (VEGF) after intravenous injection is only $50 \mathrm{~min}$, while that of FGF-2 is merely 3 min. ${ }^{[5]}$ This has led to the requirement of a regimen of repeated systemic administration at supraphysiologic doses, in order to achieve sustained effective local concentrations. ${ }^{[11]}$ This, in turn, can lead to an array of potentially complicated off-target side effects. For example, the infuse bone graft substitute (Medtronic, Memphis, TN), a collagen sponge containing recombinant human BMP-2, is indicated for use in surgical procedures for lumbar spinal fusion. However, side effects, such as cervical spine swelling, nerve root compression, ectopic bone formation, bladder retention, or hematoma, have been identified by FDA to be associated with its off-label use in other orthopedical applications. ${ }^{[12,13]}$ Similar side effects have been described for the use of Osigraft, an orthopedic implant containing BMP-7 indicated for the treatment of tibial nonunion, which has recently been withdrawn from the market. ${ }^{[13,14]}$

Furthermore, the production of the amounts required for this type of therapy currently lacks cost-effective methods, mainly due to poor yield and difficult purification, making the final costs prohibitive. ${ }^{[15]}$ These issues mostly stem from the nature of GFs as proteins with potent biological effects. This characteristic, which made them attractive in the biomedical field, also makes them dangerous molecules (notably, they play important roles in cancer progression). ${ }^{[16]}$ This means their action in physiological conditions must be tightly regulated, both spatially and temporally, through a variety of mechanisms beyond the mere regulation of gene and protein expressions, as described in the following sections. GF production and secretion can be constitutive or stimuli responsive depending on the specific molecule and cell type. ${ }^{[17]}$ They are often released in a pro-protein form, some of which have independent activity, while others need to be cleaved in order to induce a biological effect. ${ }^{[18,19]}$ Furthermore, the microenvironment plays a key role in this regulatory process.

The extracellular matrix (ECM) is a self-assembled network similar to a hydrogel, composed of a multiplicity of soluble and insoluble molecules. It provides not only mechanical support and adhesion sites for cell anchorage, but also a rich aqueous environment for biochemical interactions. ${ }^{[20]}$ Its role in biological signaling processes has been increasingly recognized. For example, cells are able to sense the mechanical properties and the spatial organization of the fibrillar microenvironment (ECM topology) via integrin-mediated mechanotransduction pathways. ${ }^{[21]}$ Another example of the role of the ECM

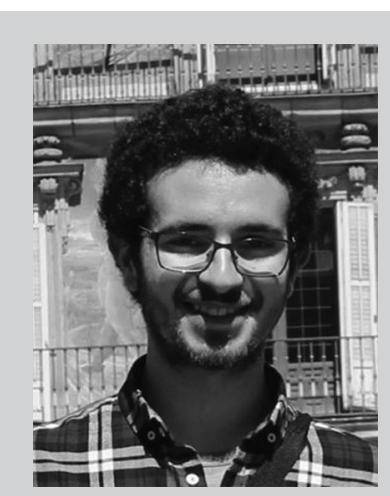

Simão P. B. Teixeira has been a Ph.D. student in tissue engineering, regenerative medicine, and stem cells at I3Bs-Research Institute for Biomaterials, Biodegradables and Biomimetics, University of Minho, since 2018. He received his M.S. degree in molecular biotechnology from the University of Porto in 2017. Teixeira's current doctoral work focuses on the use of molecular imprinting technologies for control of stem cell fate, particularly for tendon tissue engineering and regeneration.

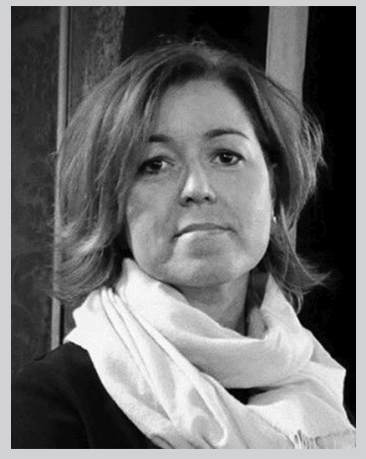

Manuela E. Gomes graduated in metallurgical and materials engineering from the University of Porto, obtained an M.Sc. in polymer engineering, and a Ph.D. in materials science and engineering-tissue engineering/hybrid materials from the University of Minho in collaboration with Rice University (USA). Presently she is Associate Professor and President of the Research Institute on Biomaterials, Biodegradables and Biomimetics (I3Bs) of the University of Minho, Portugal.

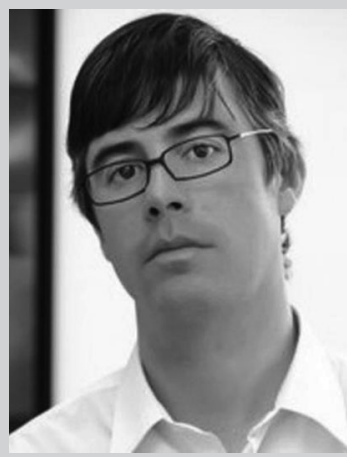

Rui L. Reis is Full Professor of Tissue Engineering, Regenerative Medicine, Biomaterials and Stem Cells at University of Minho (UMinho). He is also the Director of the 3B's Research Group of UMinho, Portugal. His group has been working on the development of biomaterials from natural polymers for engineering the regeneration of several tissues, as well as tissue engineering approaches for different 3D disease models.

is the ability of its insoluble components to bind and retain soluble molecules like GFs, dynamically regulating their release, activation, and presentation to cell surface receptors. ${ }^{[22]}$ Glycosaminoglycans (GAGs, e.g., heparin), their proteoglycan derivatives, and glycoproteins (e.g., fibronectin (FN) and laminin) are key mediators in this process. This is mainly driven by the negatively charged sulfate groups widely present on their sugar monomers, which can establish strong electrostatic interactions with many soluble factors. ${ }^{[23]}$ GAGs consist of repeating 
disaccharide units composed of an $N$-acetylated or $N$-sulfated hexosamine and either an uronic acid (GlcA or IdoA) or galactose. Hyaluronan lacks sulfate groups, but the remaining GAGs contain sulfates at various positions. ${ }^{[2]}$ Some glycoproteins also contain binding domains for several other ECM molecules, GFs, and integrins, providing another mechanism to sequester and present the soluble GFs. ${ }^{[20]}$ The context of this process is defined by whether the sequestering moiety is soluble or insoluble, its location relative to the cell, the source of the GF, and the affinity between the GF and the sequestering moiety. ${ }^{[25]}$

A good example of the complexity of regulatory processes is the different ways in which cells respond to soluble versus immobilized GFs, possibly derived from different interactions between these molecules and cell surface receptors. While soluble factors are recognized and internalized by cells as a substrate-receptor complex, immobilized factors cannot be endocytosed. This results in a sustained intracellular signaling and, therefore, in a distinct phenomenon termed "artificial juxtacrine signaling."[26] On the other hand, when GFs are sequestered by other soluble molecules, their interaction with cell receptors is instead hampered, decreasing their biological activity. This intricate interplay between the various elements that compose the cell milieu has also been shown to regulate the formation of GF gradients. For example, in the particular case of VEGF, gradients are essential to spatially and temporally direct cell migration and angiogenic sprouting. ${ }^{[25]}$

Another important point on GF activity is the crosstalk between GF receptors and integrins. ${ }^{[27]}$ Integrins are cell surface receptors, well known for their binding to ECM structural molecules with specifically defined peptide sequences, arginine-glycine-aspartate (RGD) being the most widely recognized one. ${ }^{[28]}$ It has been increasingly suggested that both direct and indirect interactions between these two types of receptors are important for the action of GFs on cellular responses. ${ }^{[29]}$ For example, syndecans-1 and -4 capture human EGF receptor 2 (HER2) and EGF receptor (EGFR), along with $\alpha_{3} \beta_{1}$ integrin, via docking sites in their ectodomains. This interaction organizes integrins and receptor tyrosine kinases into signaling complexes that stimulate epithelial invasion, contributing to tumor progression. ${ }^{[30]}$ Interestingly, it has been shown that even without the presence of their ligands, GF receptors can be directly activated by integrins via tyrosine phosphorylation, with subsequent induction of the extracellular signal-regulated kinase $1 /$ mitogen-activated protein kinase pathway. ${ }^{[31]}$

This demonstrates that ECM regulation of biological signaling is complex and related to structural, biophysical, and biochemical properties. Thus, it offers inspiration for a wide range of tunable cues that can be optimized for GF presentation in biomaterial development. In both laboratory protocols for stem cell culture and tissue engineering strategies, research efforts have progressed from direct soluble administration of GFs toward making use of materials as "carriers" for controlled release. More recently, increasing attention has been paid to the engineering of platforms that can actively and dynamically regulate biomolecular activity via noncovalent ECM-inspired interactions. ${ }^{[32]}$ This review will critically explore the main strategies used for improving the administration and regulating the activity of GFs (Figure 1).

It will particularly focus on biomaterial design approaches to sequester and retain these molecules via bioinspired affinity interactions. Specifically, these will be divided between materials that incorporate molecules naturally present in the ECM (with promiscuous interactions) and those which include specific binding motifs that recognize a target GF (such as peptides and aptamers). Each will be illustrated by some of the most representative studies on the topic, focusing particularly on those published during the last 5 years, along with their major advantages and shortcomings.

\section{Conventional Controlled Release Systems}

As previously mentioned, the limitations of soluble administration of exogenous GFs led researchers to seek biomaterial and nanotechnology approaches for their controlled delivery. These strategies sought to improve the efficiency of GF action on cells by enabling localized administration, reducing fluctuations in GF levels over time, achieving persistence of cell activating signals, and addressing diffusion limitations to soluble delivery. ${ }^{[15]}$ Conventional controlled release strategies rely on the delivery of exogenously loaded GFs through a 3D matrix or porous scaffold. This allows us to modulate the activity and differentiation patterns of stem cells through the selection of proper temporal expression and dosage of GFs. ${ }^{[3]}$ Table 1 shows a summary of GFs commonly used for some tissue engineering applications. It is important to understand the physicochemical properties, such as molecular weight, isoelectric point, and site of action when designing a vehicle for GF delivery. ${ }^{[33]}$

The most straightforward method for incorporating factors within a matrix is by noncovalent binding, which can include physical adsorption or ionic complexation. Physical adsorption is achieved by incubating the scaffold in a protein solution, allowing the formation of secondary interactions between the protein and matrix. ${ }^{[34]}$ Growth factor retention in the matrix can be increased by altering the physical and chemical properties of the biomaterial, such as mesh size, crosslink functionality, porosity, hydrophobicity, and ionic charge. ${ }^{[11]}$

Electrostatic attraction between the scaffold material and the protein has also been used to enhance the loading efficiency through the formation of polyionic complexes. For example, the processing conditions of gelatin can be modified to yield either a cationic or anionic hydrogel, which are selected based on the charge of the protein of interest. ${ }^{[35]}$ Most GFs have a high isoelectric point, indicating a positive charge at physiological $\mathrm{pH}$. Thus, anionic carriers are often used to enhance loading and achieve sustained delivery. ${ }^{[33]}$ In addition, the ionic strength of the solution or presence of other proteins can affect the ability of a GF to adsorb to the surface. ${ }^{[36]}$ While physical adsorption is often the simplest method for GF loading, it results in poorly controlled release kinetics due to a diffusiondependent, rapid burst release of the protein. ${ }^{[37]}$

Alternatively, GFs can be loaded into a matrix through physical entrapment by forming the scaffold in the presence of the protein. This technique is often used with degradable scaffolds or hydrogels, as the protein release can be controlled by the degradation or swelling of the matrix. Several techniques have been used to encapsulate GFs in the scaffold including electrospinning, ${ }^{[38,39]}$ gas foaming/particle leaching, ${ }^{[40]}$ and in situ polymerization. ${ }^{[41]}$ The release profile of GFs encapsulated 


\section{Conventional controlled} release systems

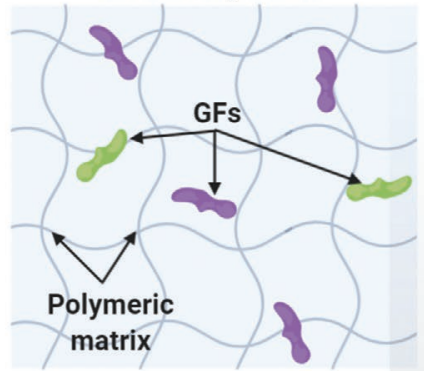

Platforms with inclusion of ECM components
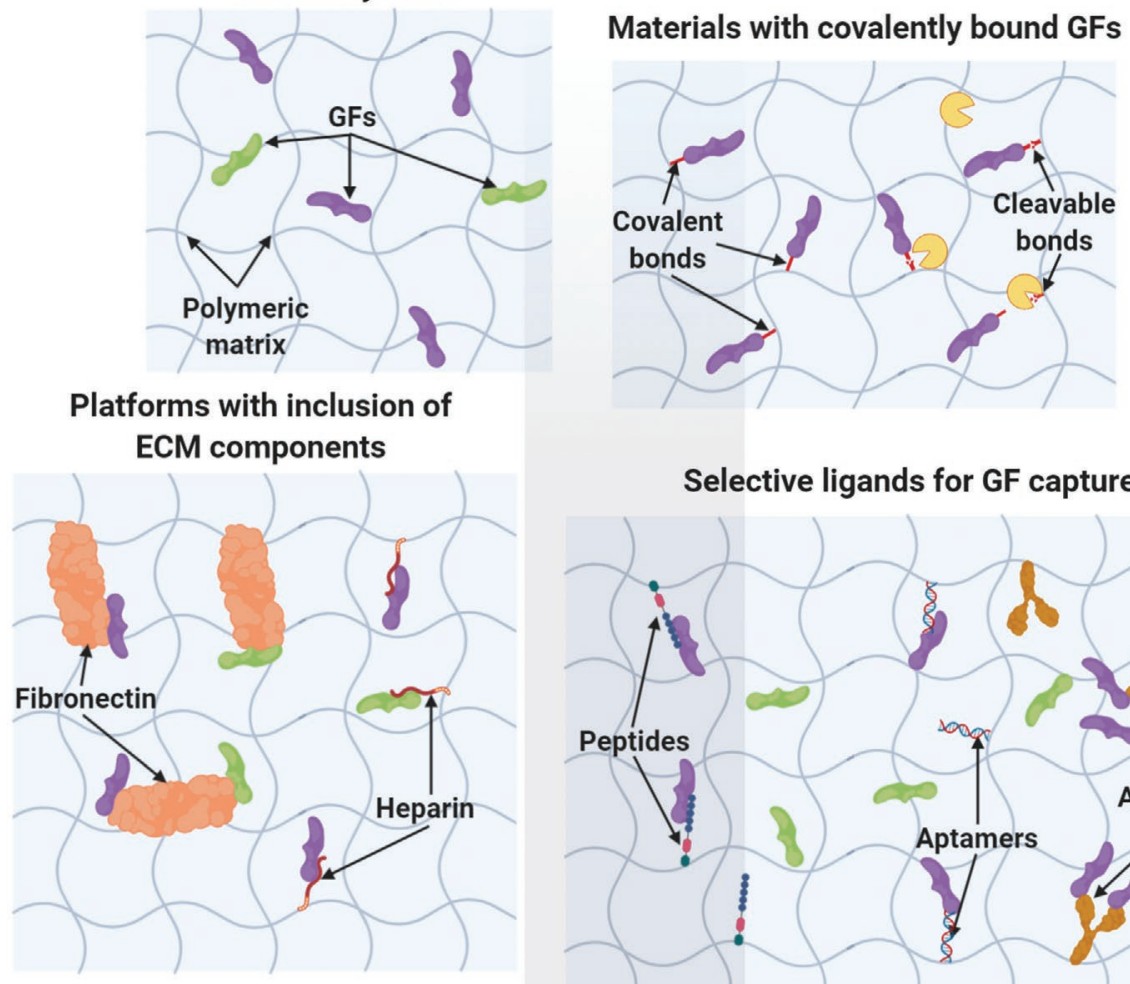

Selective ligands for GF capture

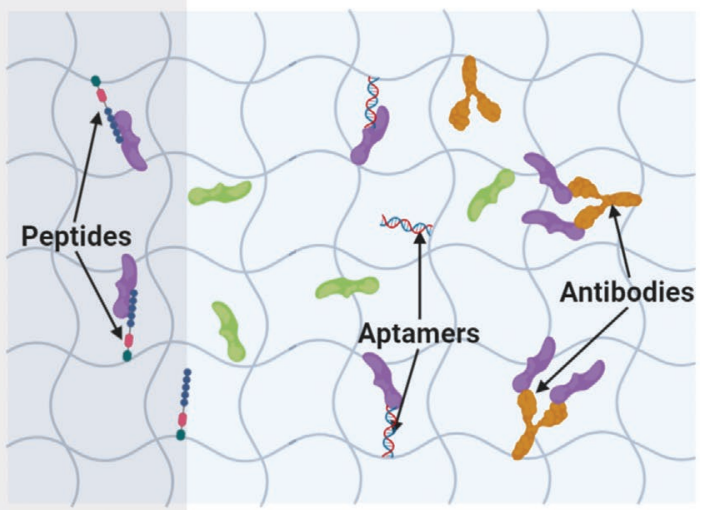

Next-generation synthetic platforms with abiotic affinity

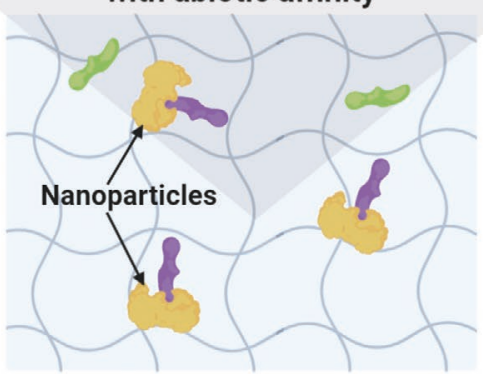

Figure 1. Overview of the main strategies for biomaterial-scaffold functionalization with growth factors and their binding partners. Conventional controlled release systems rely on the physical adsorption or entrapment of GFs in a carrier biomaterial. Alternatively, GFs may be covalently bound to the carrier material, leading to prolonged retention. These covalent bonds can include cleavable motifs to allow a programmed release according to selected stimuli. More recently developed platforms include affinity-binding molecules. These can be based either on natural ECM components, which promiscuously interact with a variety of GFs or on ligands which selectively bind a target molecule. Finally, novel synthetic platforms with abiotic affinity are envisioned to constitute the next-generation of affinity-binding systems, by allowing a less costly and larger scale production.

using these techniques will depend on whether the polymer matrix undergoes surface erosion or bulk erosion. In surface eroding polymers, release behavior is maintained over time, whereas in bulk eroding polymers, the diffusivity of the GFs within the matrix will change as the polymer degrades, resulting in a variable release profile. ${ }^{[33]}$ While selection of appropriate polymers can allow for control over GF release, one major disadvantage for physical encapsulation of GFs in the scaffold is a potential for loss of bioactivity due to harsh processing conditions. ${ }^{[34]}$

Both synthetic and natural biomaterials have been explored as polymeric matrices for encapsulating GFs and controlling their release. Synthetic polymers are widely available and offer a finer degree of control and tuning over properties, as well as presenting negligible immunological concerns compared to polymers of natural origin. ${ }^{[37,42]}$ Polyethylene glycol (PEG), polycaprolactone (PCL), ${ }^{[43]}$ poly(lactic acid) (PLA), ${ }^{[1]}$ poly(lactic-co-glycolic acid) (PLGA), ${ }^{[44]}$ polydopamine (PDA), ${ }^{[45]}$ and polyphosphazene ${ }^{[46]}$ all constitute examples of biocompatible polymers tested in recent years for controlled GF release systems. On the downside, synthetic polymers are not endowed with the bioactivity that natural polymers can offer, particularly those derived from ECM components possessing cell and biological factor interactive domains. ${ }^{[37]}$ These properties have 
Table 1. Physicochemical properties of common growth factors for cartilage, bone, and neural tissue engineering. Molecular weight and isoelectric point measurements taken from the cited literature or calculated using the ExPASy bioinformatics resource portal. Acronyms: Nerve growth factor (NGF), brain-derived neurotrophic factor (BDNF), neurotrophin-3 (NT-3), glial cell line-derived neurotrophic factor (GDNF), ciliary neurotrophic factor (CNTF), fibroblast growth factor (FGF), and placental growth factor (PLGF). Reproduced with permission. ${ }^{[33]}$ Copyright 2018, Elsevier.

\begin{tabular}{|c|c|c|c|c|c|}
\hline Family & Name & $\mathrm{MW}[\mathrm{kDa}]$ & $\mathrm{pl}$ & $\begin{array}{c}\text { Site of action } \\
(\mathrm{E}=\text { Extracellular }, \mathrm{C}=\text { cytoplasm })\end{array}$ & Common use \\
\hline \multirow[t]{3}{*}{ Transforming growth factors (TGF) } & TGF- $\alpha 1$ & 5.56 & 5.9 & EGF receptor $(E)$ & $\begin{array}{l}\text { Angiogenesis, promotion of cell } \\
\text { migration or proliferation }\end{array}$ \\
\hline & TGF- $\beta 1$ & 25 & 9.5 & TGF- $\beta 1$ receptor $(\mathrm{E})$ & $\begin{array}{l}\text { Chondrogenic differentiation, } \\
\text { increase adhesion molecule expression }\end{array}$ \\
\hline & TGF- $\beta 3$ & 25.8 & 8.3 & TGF- $\beta 2$ receptor (E) & $\begin{array}{l}\text { Chondrogenic differentiation, } \\
\text { promote proliferation }\end{array}$ \\
\hline \multirow[t]{2}{*}{ Insulin-like growth factors (IGFs) } & IGF-1 & 7.6 & 7.8 & IGF-1 receptor $(E)$, insulin receptor $(E)$ & $\begin{array}{l}\text { Cartilage homeostasis, } \\
\text { chondrogenesis }\end{array}$ \\
\hline & IGF-2 & 7.5 & 6.4 & IGF-II receptor $(E)$, insulin receptor $(E)$ & $\begin{array}{c}\text { Osteogenic and chondrogenic } \\
\text { differentiation }\end{array}$ \\
\hline \multirow[t]{2}{*}{ Bone morphogenetic proteins (BMP) } & BMP-2 & 12.9 & 8.2 & BMP receptor type II (E) & $\begin{array}{l}\text { Osteogenic differentiation } \\
\text { and osteogenesis }\end{array}$ \\
\hline & BMP-7 & 15.7 & 8.1 & BMP receptor type II (E) & Chondrogenesis, osteogenesis \\
\hline \multirow[t]{3}{*}{ Neurotrophins } & NGF & 32.5 & 9.3 & TrkA receptor, p75 (E) & $\begin{array}{l}\text { Survival and outgrowth } \\
\text { and branching of sensory } \\
\text { neurons, nerve regeneration }\end{array}$ \\
\hline & BDNF & 13.5 & 9.6 & TrkB receptor, p75 (E) & $\begin{array}{l}\text { Motor and sensory neuron } \\
\text { outgrowth and survival }\end{array}$ \\
\hline & NT-3 & 13.6 & 9.2 & TrkA, TrkB, TrkC, p75 receptors (E) & Neuron outgrowth, nerve regeneration \\
\hline \multirow{2}{*}{$\begin{array}{l}\text { Vascular endothelial growth factor } \\
\text { (VEGF) }\end{array}$} & VEGF-A & 23.9 & 9.2 & VEGFR-1 and VEGFR-2 (E) & Angiogenesis \\
\hline & PLGF & 22.8 & 8.4 & FLT1/VEGFR-1 receptor (E) & VEGF stimulation, Angiogenesis \\
\hline
\end{tabular}

stimulated the use of materials based, for example, on hyaluronic acid (HA), ${ }^{[47,48]}$ collagen type $\mathrm{I},{ }^{[49]}$ or gelatin, a product of collagen hydrolysis. ${ }^{[50-53]}$ Besides ECM molecules, other natural polymers widely researched in the field of tissue engineering have also been studied recently for GF delivery. Among these are alginate, a polysaccharide extracted from the cell wall of brown seaweed that grows in cold water regions, ${ }^{[54-56]}$ chitosan, another polysaccharide, produced by controlled deacetylation of chitin, ${ }^{[57]}$ and silk fibroin (SF), one of the two main constituent proteins in silk produced by certain arthropods. ${ }^{[58]}$ Alginate exhibits excellent biocompatibility and biodegradability, as well as an ability to be readily processed for 3D scaffolding materials. ${ }^{[59]}$ Chitosan is one of the few natural polymers with a polycationic nature, providing good adhesive properties and high biocompatibility, ${ }^{[60]}$ while silk fibroin presents low immunogenicity, high mechanical strength, and controllable degradation behavior. ${ }^{[61]}$ However, unlike ECM-derived molecules these do not provide biological interactive motifs, requiring combination with other materials or immobilization of specific ligands to effectively control cellular responses. ${ }^{[59-61]}$

Depending on the target application, these systems can be processed into nanocapsules, fibers, layer-by-layer (LbL) assemblies, or hydrogels, among others. Nanocapsules, for instance, allow the loading of GFs, shielding them from the surrounding environment. Furthermore, release rates can be tuned by engineering the polymer shell composition. For example, a matrix metalloproteinase (MMP)-sensitive peptide has been used as crosslinker in 2-(methacryloyloxy)ethyl phosphorylcholine (MPC)-based nanocapsules for the delivery of BMP-2 to promote bone regeneration. ${ }^{[10]}$ Since MMPs' secretion is upregulated at the injury site during bone repair process, GF release triggering could be synchronized with tissue remodeling. On the other hand, glycerol dimethacrylate (GDMA) was used in a different study as crosslinker for similar polymeric capsules. ${ }^{[62]}$ Since GDMA degrades faster in the relatively alkaline environments' characteristic of the later stages of bone healing, these nanocapsules present a healing phase-dependent degradation, enabling a sustained release of encapsulated GFs at the target site (Figure 2b). Synthetic polymer fibers have also been used for loading GFs for prolonged release. ${ }^{[39]}$ In an example of this strategy, polydioxanone fibers commonly used in surgical sutures were loaded with VEGF to promote neovascularization, ensuring adequate blood supply and cell survival in endodontic procedures. ${ }^{[63]}$

LbL constructs rely on a stacking of two or more types of polymers, usually achieved by using materials with opposite charge. ${ }^{[64]}$ These materials allow the loading of various bioactive molecules, including GFs, which can then be released in a temporally controlled manner depending on the layer they are incorporated in. ${ }^{[64,65]}$ Furthermore, this technique is highly versatile not only in terms of geometry-it has been applied to planar surfaces, spherical particles, inside pores, and onto other more complex material geometries-but also in chemistry, since it can be combined with a variety of surfaces, including noncharged 
a
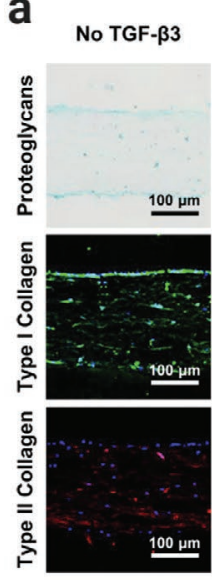

Low-Dos TGF- $\beta 3$
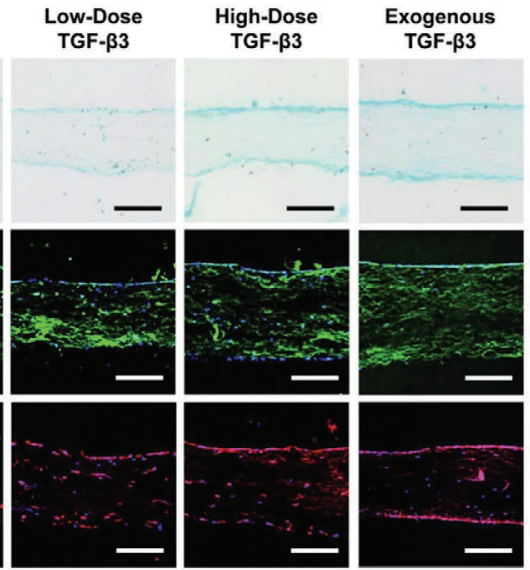

C
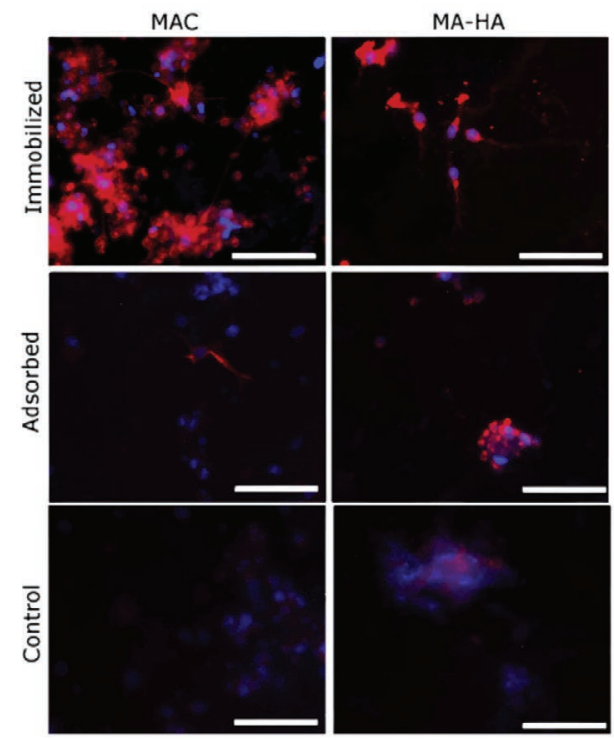

b
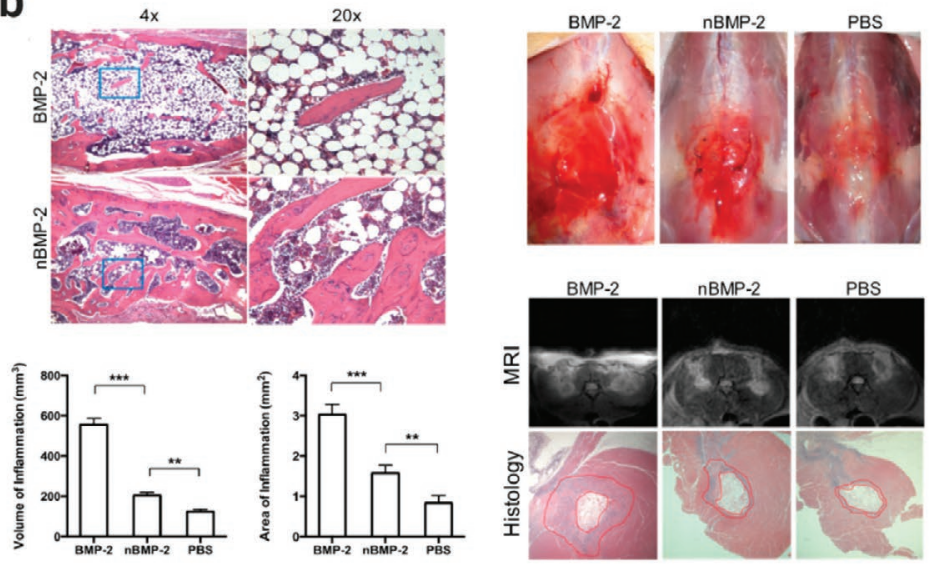

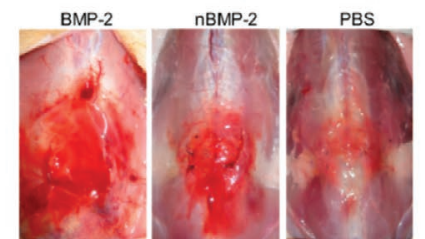

d

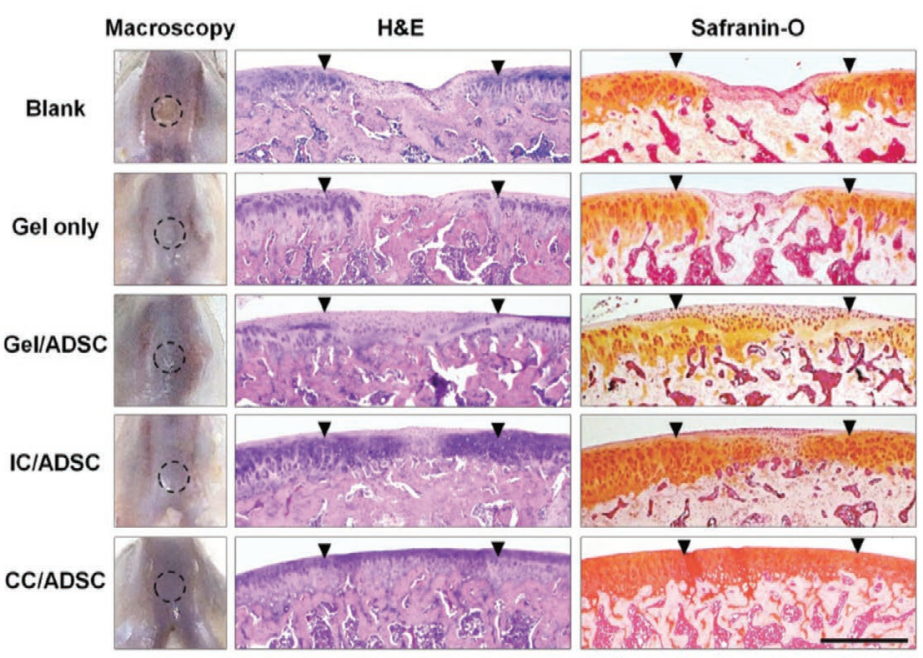

Figure 2. a,b) Controlled release and c,d) covalent binding of GFs to carrier biomaterials. a) Synthesis of fibrocartilage-like matrix by synovium-derived stem cells cultured on PCL-PLGA electrospun nanofibers encapsulating TGF- $\beta 3$. Synthesized collagen matrix followed the alignment of underlying scaffold. Proteoglycan deposition (Alcian blue staining) was higher in high-dose TGF- $\beta 3$ experimental group compared to the low-dose groups and groups with no TGF- $\beta 3$, although not at the same level as the exogenous supplementation group. Adapted with permission. ${ }^{[43]}$ Copyright 2019 , Elsevier. b) In vivo evaluation of nano-encapsulated BMP-2. Nano-encapsulated BMP-2 promoted formation of more trabecular bone (upper left), while leading to a tamer inflammatory reaction (right side). Adapted with permission. ${ }^{[62]}$ Copyright 2016, American Chemical Society. c) Immobilized azide-tagged IFN- $\gamma$ promotes greater neuronal differentiation of NSCs. $\beta$ III-tubulin immunostaining shows more pronounced neuronal phenotype and longer neurite outgrowth for cells cultured on HA-based hydrogels with immobilized protein, compared to simple adsorption. Adapted with permission. ${ }^{\text {[7] }}$ Copyright 2017, Elsevier. d) Histological analysis of cartilage defects created in rat knee trochlear cartilage. Rats treated with MeGC/Col II hydrogels with covalently conjugated TGF- $\beta 1$ and encapsulated ASCs (CC/ADSC) show remarkable recovery compared to other conditions, namely encapsulated ASCs without the GF (Gel/ADSC) and encapsulated ASCs in gels with ionic conjugated TGF- $\beta 1$. Adapted with permission. ${ }^{\text {[2] }}$ Copyright 2015 , The Royal Society of Chemistry.

substrates. ${ }^{[66]}$ For example, this strategy has been successfully applied to produce osteoinductive scaffolds that could first release a burst of the proangiogenic connective tissue growth factor (CTGF), followed by a sustained release of pro-osteogenic BMP-2. ${ }^{[67]}$ Another application of LbL is in implant coating to improve their biological integration with host tissues. For example, 3D PLGA tubes were used as polymeric scaffolds for structural and mechanical supports for long bone regeneration. Poly-L-lysine/HA polyelectrolyte film coatings were applied on the tubes in order to load BMP-2 and tune its release. ${ }^{[68]}$
The system achieved an effective and fast repair in 1-2 weeks, completely bridging a critical sized femoral defect in a rat model. A different study used multilayered coatings, composed of polymers with different degradation rates, to sequentially deliver two therapeutic agents and improve the integration of bone implants. ${ }^{[69]}$ First, a fast release of the antibiotic gentamycin cleared established bacterial biofilms. Next, sustained release of BMP-2 for several weeks promoted the regeneration of the previously infected bone surface, improving bone-implant interfacial strength 15-fold when compared with uncoated implants. 
However, hydrogels constitute the preferred delivery vehicles due to their ECM-mimicking structure and possibility of in situ gelation for injectable administration. In bulk form, they have been applied, e.g., in the regeneration of vocal fold mucosa by gradually releasing hepatocyte growth factor $(\mathrm{HGF})^{[41]}$ and FGF-2, ${ }^{[51]}$ or for cardiac repair after myocardial infarction, by releasing citrate and myeloid-derived growth factor. ${ }^{[70]}$ In microspheres, hydrogels have been tested, for example, to steer the osteogenic commitment of mesenchymal stem/stromal cells (MSCs) by release of BMP-2. ${ }^{[52]}$

A recent representative example of this type of systems is the combination of unmodified alginate with alginate sulfate, which presents an increased negative charge conferring higher affinity to multiple GFs, to develop an ovary mimetic microenvironment. ${ }^{[56]}$ This system could retain BMP-4 by electrostatic interactions and, in turn, support the culture and growth of porcine primordial follicles seeded with supporting ovarian cells. These scaffolds were able to sustain the development of the follicles up to the preantral stage, with five times more developing follicles and estrogen production than the groups with soluble BMP-4. More significant still is the ability of these devices to restore ovarian function after xenotransplantation in ovariectomized severe combined immunodeficiency (SCID) mice, providing the first successful example of GF presentation for this purpose. ${ }^{[56]}$

Despite the continued improvements in this type of controlled release systems, there are various aspects of previously described GF signaling mechanisms that are not taken into consideration. They constitute an advanced delivery system for recombinant versions of GFs, allowing a more sustained and prolonged release to circumvent some problems of soluble bolus administration. However, the reliance on physical entrapment and diffusion mechanisms makes it difficult to tune release behavior, particularly when considering multiple molecules in a single application. Moreover, the multiple effects resulting from the microenvironmental regulation of GF action, such as sequestering and solid-phase presentation, are generally not present. These have been shown to be preferable for eliciting several cell responses, including the possibility of GF receptor-integrin crosstalk. ${ }^{[22,27]}$

\section{Solid-Phase Presentation}

In order to more closely mimic the mode of action of GFs in natural biological microenvironments and potentiate their activity (thereby also reducing the therapeutic doses and associated costs), several methods to prepare scaffolding biomaterials with the ability to present solid-phase-bound GFs have been increasingly studied. They can be broadly divided into two groups depending on if GFs are covalently bound to the delivery vehicle or if the latter is designed for increased affinity, with the ability to sequester and noncovalently bind and retain GFs.

\subsection{Materials with Covalently Bound GFs}

The covalent binding of GFs to a supporting biomaterial presents several advantages over previously discussed alternatives.
First, the proteins do not diffuse into the surrounding medium over time. Combined with the amplification of GF activity resulting from their solid-phase presentation (see Section 1), this allows the use of even lower doses to achieve similar biological effects. Second, the tethering of proteins to a solid support renders them less susceptible to hydrolytic and enzymatic degradation, helping to prolong their activity. Moreover, it is possible to achieve more precise patterning and spatial organization of GFs in these systems. ${ }^{[5,26]}$ A variety of conjugation strategies and reactions can be used for this purpose, which have been reviewed in detail elsewhere. ${ }^{[26,73]}$ Some of the most recent reports on the subject have successfully bound GFs on several polymeric biomaterials, including stem cell factor $(\mathrm{SCF}),{ }^{[74}$ transforming growth factor beta 1 (TGF- $\left.\beta 1\right),{ }^{[72,75]}$ EGF, ${ }^{\left[{ }^{76-79]}\right.}$ FGF-2, ${ }^{[76]}$ nerve growth factor (NGF), ${ }^{[80]}$ or VEGF. ${ }^{[81]}$

Modification of gelatin with methacryloyl moieties, known as GelMA, allows the application of mild photopolymerization techniques for crosslinking. Photo-crosslinking minimizes the use of toxic agents or aggressive conditions which could damage or compromise protein stability. Furthermore, this modification can also be leveraged for the covalent linkage of GFs, as shown by Mahadik et al. ${ }^{[7]}$ In this work, acrylatefunctionalized PEG was used to tether SCF to the GelMA hydrogel, achieving stable incorporation and retention over a week. While soluble SCF induces increased proliferation of differentiating hematopoietic stem cells (HSCs), this system led to opposite effects. Cultured primary HSCs showed reduced proliferation and improved selectivity for maintaining a primitive phenotype. This system was then combined with microfluidics to generate SCF immobilization gradients capable of spatially directing stem cell responses. ${ }^{[7]}$ This further illustrates the differences between soluble and solidphase-presented GFs, and how both can be selectively and rationally applied for stem cell culture procedures.

Cartilage tissue is mostly avascular and has limited healing potential after serious injury or prolonged disease. ${ }^{[82]}$ TGF$\beta 1$ is well known for its important role in chondrogenesis. It has thus been explored to stimulate regeneration in damaged cartilage, in conjunction with both synthetic and natural polymeric biomaterials. ${ }^{\left[{ }^{[2]}\right.}$ For example, succinimidyl-4-( $N$-maleimidomethyl)cyclohexane-1-carboxylate (SMCC) was used to link TGF- $\beta 1$ to methacrylate glycidyl chitosan (MeGC), mixed with collagen type II (Col II), the most prevalent collagen type in cartilage tissue. Irradiation with visible blue light was used to crosslink the mixture. ${ }^{[72]}$ Encapsulated adipose-derived stem cells (ASCs) were able to deposit more cartilaginous ECM. Furthermore, robust cartilage regeneration was observed when implanted in a rat partial-thickness chondral defect model (Figure 2d). ${ }^{[72]}$

In another study, PEG hydrogels were produced through a photoinitiated step-growth polymerization, by reacting norbornene-terminated PEG macromolecules with a dithiol PEG crosslinker. ${ }^{[75]}$ Thiolated TGF- $\beta 1$ was included in this system and conjugated to PEG-norbornene by thiol-ene click reaction. Compared to TGF- $\beta 1$ delivered by solubilization, chondrocytes encapsulated in hydrogels with tethered TGF- $\beta 1$ showed significantly increased DNA content, GAG, and collagen production over 28 days, while maintaining markers of articular cartilage. ${ }^{[75]}$ 
As exemplified above, thiol chemistry is a biocompatible and relatively efficient way to link GFs to solid-phase supports by taking advantage of existing cysteines in the protein. Several groups explored it to conjugate VEGF to scaffolding biomaterials designed to promote vasculogenesis, a key process to increase graft survival. ${ }^{[81,83]}$ For example, VEGF was conjugated to a maleimide-functionalized PEG hydrogel to improve the survival of transplanted pancreatic islet grafts. ${ }^{[83]}$ Among the tested host sites, epididymal fat pads showed the most vascularization and least inflammation. Overall, this strategy could successfully improve survival, engraftment, and function of a single pancreatic donor islet graft compared to the current clinical intraportal delivery technique. Thus, this study demonstrated potential to improve the lives of insulin-dependent diabetic patients. ${ }^{[83]}$

In a somewhat different approach, poly(vinyl alcohol) (PVA) hydrogels were synthesized by reversible addition-fragmentation chain transfer (RAFT) polymerization, with terminal thiol groups being activated toward disulfide exchange. ${ }^{[76]}$ A commercial protein cocktail containing mainly EGF and FGF-2 could then be successfully coupled to these moieties. GF-containing gels could communicate to adherent cells, and specifically enhanced proliferation of cells with the corresponding receptors (human umbilical vein endothelial cells, HUVECs), while exerting no specific effects on other cell types (myoblasts). ${ }^{\text {76] }}$

Another highly biocompatible conjugation alternative is the use of click chemistry, particularly copper-free reactions. For example, strain-promoted azide-alkyne cycloaddition (SPAAC) has been used to couple azide-modified EGF to primary amines in collagen, by means of dibenzo cyclooctyne (DBCO)-sulfo- $N$-hydroxysuccinimide (NHS) ester. ${ }^{[78]}$ This is a fast, catalyst-free, bio-orthogonal click reaction that proceeds to stoichiometric completion under physiologically relevant temperature and $\mathrm{pH}$ conditions. ${ }^{[84]}$ The anchoring proved to be non-cytotoxic, biocompatible, and sufficiently rapid for clinical application to enhance wound healing $(\approx 5 \mathrm{~min})$. Moreover, the surface-immobilized EGF has significant effects on epithelial cell attachment and proliferation. ${ }^{\text {78] }}$

Recombinant technology can also be used to increase the control over protein binding. This is done by introducing certain tags at specific points of the GF structure, which can then be used to couple them to a biomaterial in an oriented manner. In this way, it is possible to ensure that GFs are not randomly tethered through their receptor-binding domains, thus retaining their bioactivity. ${ }^{[71]} \mathrm{Ham}$ et al. combined recombinant technology with SPAAC to create platforms for guiding neural stem cell (NSC) behavior. ${ }^{[71]}$ To that end, a recombinant azide-tagged interferon-gamma (IFN- $\gamma$ ) was immobilized onto two different DBCO-functionalized biomimetic polysaccharides (chitosan and HA). Immobilized IFN- $\gamma$ retained its full bioactivity, significantly increasing neuronal differentiation when compared to physically adsorbed IFN- $\gamma$ (Figure $2 \mathrm{c}$ ). Differentiation into other lineages varied between chitosan and HA, once again demonstrating the complex interplay between different stimuli on the control of stem cell fate. ${ }^{[71]}$

Notwithstanding the latest advances of these systems, the strategy of covalently binding GFs to their supporting structure has raised some concerns. Conceptually, its chief aim is to avoid the "burst release" behavior often observed when
GFs were physically adsorbed onto carrier systems, as well as to mimic the juxtacrine signaling mechanisms of the natural ECM. Both problems are indeed tackled, but in nature GFs have well-orchestrated transient effects during tissue development, repair, and regeneration.

As previously mentioned, their action is tightly regulated not only spatially but also temporally, which does not happen with the covalent tethering on biomaterials (besides the degradation of the scaffold itself). It has thus been suggested that they may remain present for too long, inadvertently interfering with the regenerative and other biological processes. Furthermore, covalent immobilization inhibits GF internalization, thereby sustaining the intracellular signaling pathways by receptor binding and prolonging their downstream activity. However, not all GFs act in the same manner, and some are required to be internalized to properly exert their effects. ${ }^{[26]}$ Another associated problem is the maintenance of conformation and/or biological activity after conjugation. In this regard, the presented approaches have increasingly avoided aggressive reaction conditions that could denature the proteins. Nonetheless, it is particularly difficult to control the orientation of GFs and the sites where conjugation reactions occur. This can lead to the masking and steric hindrance of receptor-binding sites, preventing the access of GFs to their cognate receptors, and causing loss of biological activity. ${ }^{[26]}$ The production of specifically designed recombinant proteins can mitigate this limitation, but the whole process is overall more complex and expensive. ${ }^{[1]}$

In sum, this type of approach leads to the production of mostly static biomaterials, which fail to replicate the dynamic nature of GF activity and the changes happening over the course of biological processes. ${ }^{[85]}$

\subsubsection{Covalent Binding with Stimuli-Sensitive Linkers}

In order to tackle these issues and introduce dynamism in the systems, cleavable linkers have been increasingly sought. Although GFs are still covalently linked to the supporting material to increase their stability and prolong signaling activity, the linker is sensitive to a given stimulus capable of triggering its cleavage and releasing the protein into the surrounding medium. This function should ideally be designed so as to result in a controlled release system synchronized with the needs of the specific application. In this sense, this approach can be understood as a combination of the previous two, but with an additional potential for temporal and spatial dynamic control. ${ }^{[85-91]}$

A well-established way to achieve this goal is to produce recombinant proteins that include two particular domains at the N-terminus: a crosslinking site that allows coupling to the scaffold and a cleavage site that allows protein release over the course of regeneration. Both domains can be sequences sensitive to enzymes naturally present in the human body, ensuring that conjugation reaction conditions are protein-friendly, and that protein release is triggered by the protease activity of recipient tissue. Transglutaminase (TG) substrate domain is particularly attractive since it allows crosslinking by TGs such as the blood coagulation protein factor XIII (FXIII). ${ }^{[86-89]}$ FXIII is 
normally involved in the crosslinking and stabilization of fibrin networks in blood clots. ${ }^{[92]}$ By including the recombinant GF into this mixture, FXIII will also link its substrate domain to the growing fibrin matrix, immobilizing the GF onto the scaffold. Fibrin is also a well-studied material for regenerative medicine purposes, due to its inherent hemostatic and bioadhesive properties, as well as being biodegradable and of natural origin. Thus, it constitutes another alternative platform for stem cell and GF delivery. ${ }^{[86]}$ Regarding the cleavable sequence, plasmin is another common plasmatic enzyme that can be taken advantage of. Plasmin is a serine protease that cleaves several plasma proteins, among them fibrin, helping to dissolve blood clots. ${ }^{\text {[2] }}$ This dual approach has been studied, for example, for PDGF$\mathrm{AB},{ }^{[86]} \mathrm{BMP}-2,{ }^{[87]}$ and BMP-7 ${ }^{[88,89]}$ immobilization in fibrin hydrogels.

PDGF-AB is a well-known proangiogenic factor that stimulates VEGF production and recruits smooth muscle cells. These are thought to be stabilizers of blood vessels, thereby contributing to a more mature and effective vasculature. ${ }^{\left[{ }^{86]}\right.}$ Mittermayr et al. studied its inclusion in a fibrin matrix, using this recombinant approach, which could be applied by spraying for improved regeneration in a standard ischemic flap model. 7 days after ischemic insult, a single application was enough to significantly reduce tissue necrosis and shrinkage compared to a commercial fibrin sealant control. Laser Doppler imaging also revealed that vessel extension and maturation were superior in the treatment groups, along with enhanced von Willebrand factor (vWF) expression and improved smooth muscle actin/vWF ratio. ${ }^{[86]}$ This strategy has also been tested with an engineered BMP-2 for bone regeneration, with promising in vivo results in dog pancarpal fusion and rat calvarial defect models, surpassing even cancellous bone autograft. ${ }^{[8]}$ More recently, a BMP-2/7 heterodimer was evaluated in the same rat model, leading to nearly twice increased new bone formation compared to the previous homodimer. ${ }^{[88]}$ Both dimers have also been conjugated to a fibrin/HA hydrogel and injected in a goat model of mild intervertebral disc degeneration. ${ }^{[89]}$ However, neither showed significant regeneration of injured discs after 12 weeks of evaluation, regardless of the concentration or type of applied GF (homo- or heterodimer). Possible explanations pointed by the authors of the study include too low dosages, short follow-up time, and/or insufficient release of the conjugated BMPs, which should be addressed in future studies. ${ }^{[89]}$

Besides enzymatic susceptibility, novel bioconjugation reactions have been sought to allow greater spatial and temporal control over biomolecule tethering. Recent efforts have achieved remarkable degrees of spatiotemporal patterning of protein modification on hydrogel substrates, by making use of cytocompatible near-UV light $(\lambda=365 \mathrm{~nm})$ photoscission. Shadish et al. established a new enzymatic method to precisely, uniformly, and efficiently graft the desired moieties to recombinant protein C-termini. This method proved to be superior to commonly used NHS reactions in terms of retaining GF bioactivity, ease of purification, and reaction efficiency. It was subsequently used to introduce an ortho-nitrobenzyl ester (oNB) moiety which undergoes rapid photoscission upon exposure to $365 \mathrm{~nm}$ light. ${ }^{[90]}$ Mask-based multiphoton lithographic techniques were then exploited to precisely control oNB-protein release and concentration in 4D fashion. Furthermore, this technique could be applied on 2D cell cultures to attain accurate spatial regulation over A431 cell proliferation, by selectively patterning EGF-oNB$\mathrm{N}_{3} \cdot{ }^{[90]}$ Grim et al. presented a similar concept, with the additional advantage of repeatable addition of more proteins to replace the ones being released. ${ }^{[85]}$ To that end, an allyl sulfide chain transfer agent was designed to allow the reversible photomediated bioconjugation of thiolated proteins to the hydrogel backbone. The release is processed by addition of a simple PEG thiol, which also regenerates the "ene" functionality, allowing further coupling of new molecules. This system could also be used in the presence of mouse embryonic fibroblasts, which were shown to respond to the selective uncoupling of TGF- $\beta 1$, lowering their Smad expression. ${ }^{[85]}$

These cleavable linker approaches have thus made great strides is recent years, and increasingly revealed to be extremely versatile options for controlled GF delivery and presentation. They hold the advantages of GF stabilization and retention on the scaffold provided by covalent linking, while engineering their programmed release according to given stimuli. Thus, these systems can capture, to some extent, the dynamic behavior of the natural ECM, and hold great promise for the near future. However, these advances have been possible at the expense of an increasing degree of complexity and associated costs. Along with the fact that they still rely on the inclusion of recombinant GFs, and considering their previously mentioned drawbacks, it must be pondered if large-scale production is feasible and economically viable. Indeed, despite recent advances, their translation perspectives toward commercial and clinical applications may still be far off. ${ }^{[93]}$

\subsection{Materials with Increased Affinity for GFs}

In order to avoid the problems associated with chemical reactions for covalent coupling and the increasingly complex biomaterials required for controllable release, other lines of research have been developing. They have focused on platforms with increased affinity toward GFs so as to efficiently sequester these molecules from solution and present them at their surface for interaction with cells. These biomimetic strategies are generally inspired by the way ECM and GFs interact in their natural environments, where noncovalent interactions predominate.

\subsection{Platforms with Inclusion of ECM Components}

The most straightforward way to mimic the functioning of the ECM is by the use of molecules present in the ECM itself. To that effect, two main groups of molecules have been widely explored, namely sulfated GAGs-chiefly heparin-and proteins which contain binding sites for GFs and/or heparinfibronectin being the most researched one. ${ }^{[32,94-96]}$

Heparin, Its Derivatives, and Mimetics: As previously mentioned, the ECM contains a number of molecules able to sequester and regulate GF activity. Among these are GAGs containing sulfate groups, which confer a strong overall negative charge. Heparin has attracted particular attention thanks to its commercial availability and easy accessibility. It can electrostatically bind GFs, protecting them from enzymatic 
a

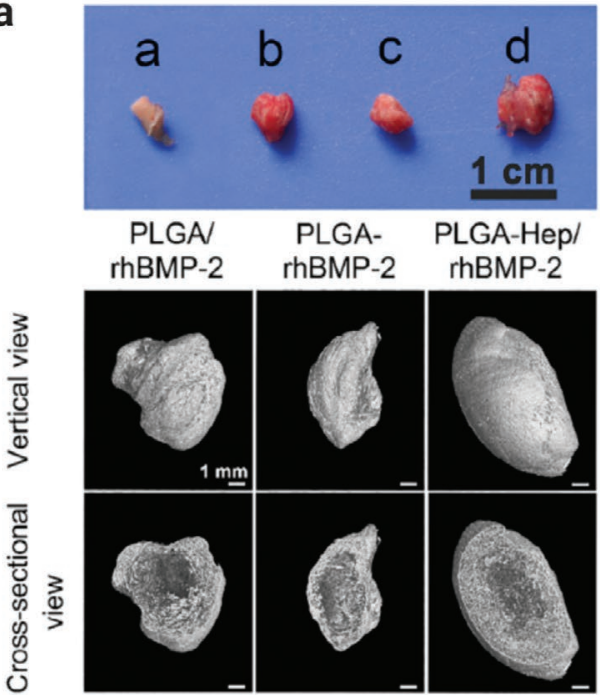

C
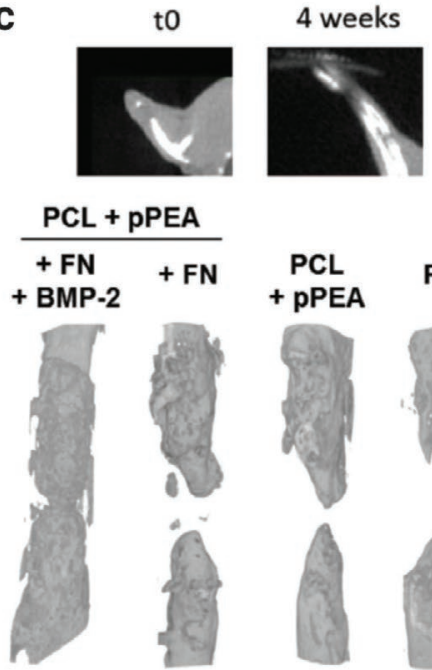

$+\mathrm{FN}+\mathrm{FN}$

BMP-2

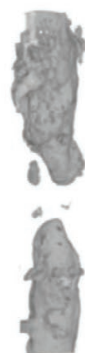

$\mathrm{PCL}+$ pPEA
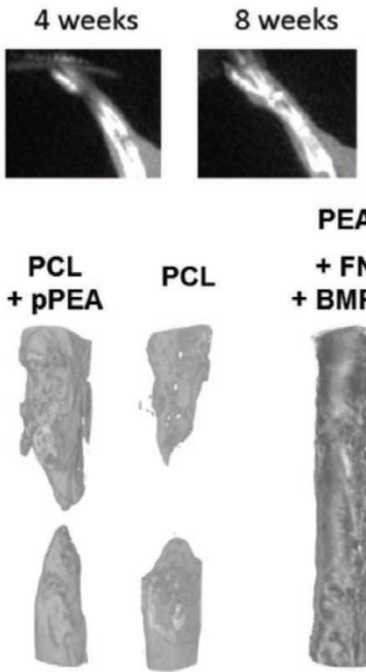

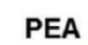

b
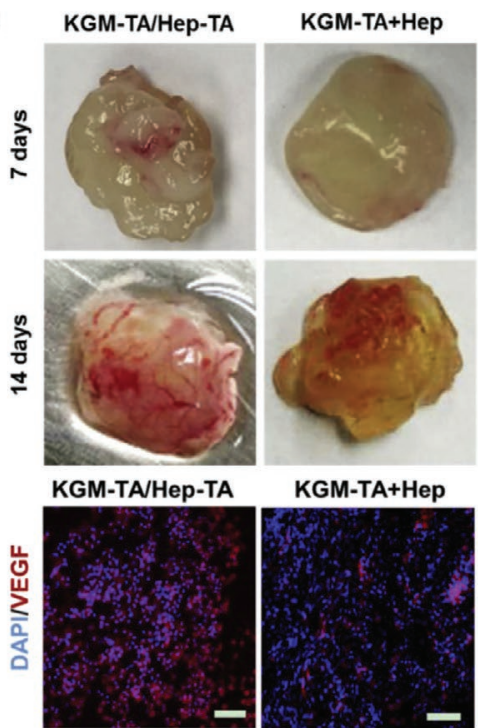

KGM-TA

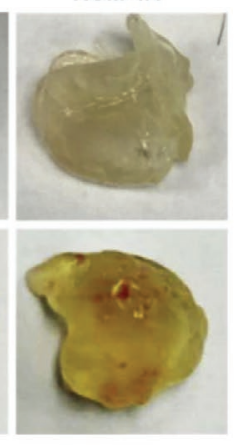

KGM-TA
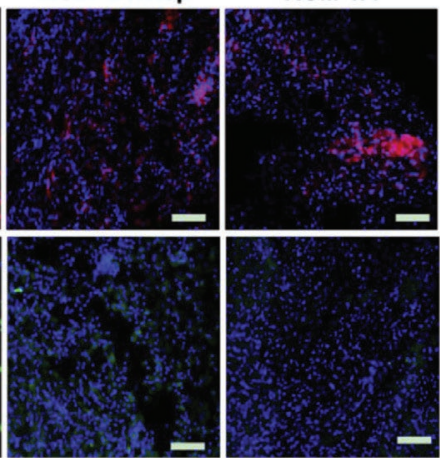

d
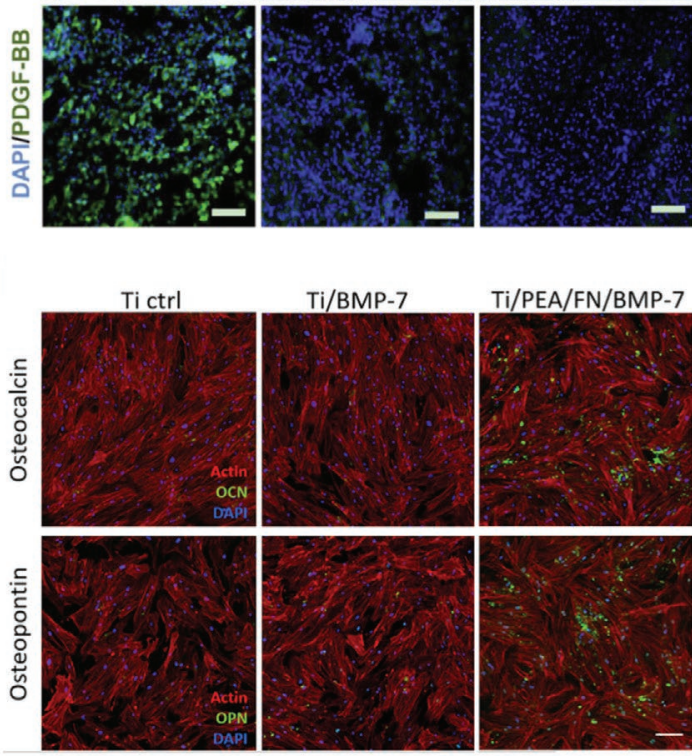

PCL

+ FN

+ BMP-2
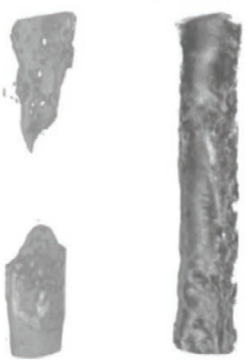

Ti/PEA/FN/BMP-7
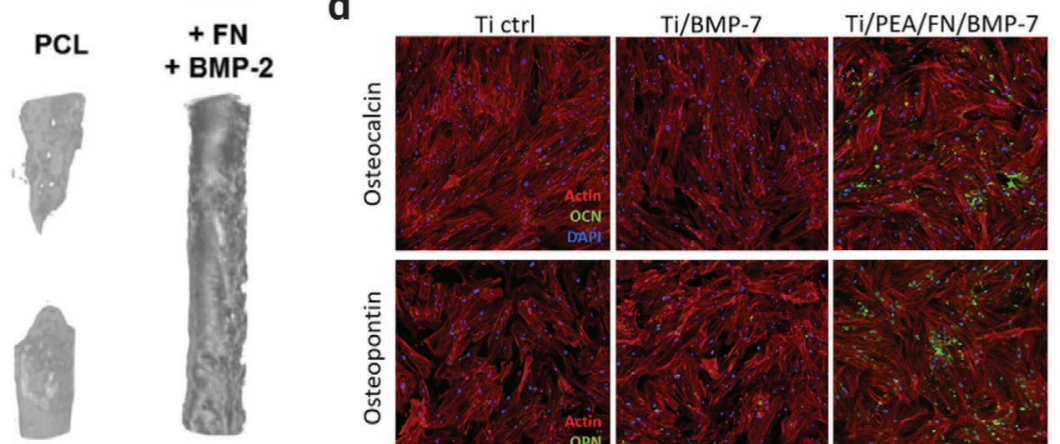

Figure 3. Biomaterial platforms with inclusion of ECM components. a) PLGA scaffolds conjugated with heparin and containing affinity-bound rhBMP-2 (PLGA-Hep/rhBMP-2 in lower panel, part (d) in upper panel) induced superior ectopic bone formation in mice at week 2, compared to scaffolds with rhBMP-2 but without heparin. Upper panel: digital photographs of explants; lower panel: 3D micro-computed tomography ( $\mu C T)$ reconstructive images of ectopic bones. Adapted with permission. ${ }^{[98]}$ Copyright 2018, Elsevier. b) In vivo sequestration of proangiogenic GFs by hydrogels injected subcutaneously in the back of mice. Konjac glucomannan (KGM) gels with tyramine (TA)-crosslinked heparin (KGM-TA/Hep-TA) sequestered significantly more endogenous VEGF and PDGF-BB than gels with physically adsorbed heparin (KGM-TA+Hep) or no heparin (KGM-TA) (lower panel, immunostaining). This corresponded to higher levels of angiogenesis (upper panel, microscopic images of gels collected after 7 and 14 days post-implantation). Adapted with permission. ${ }^{[104]}$ Copyright 2017, Elsevier. c) PCL-pPEA-FN-coated implants loaded with low doses of BMP-2 lead to formation of higher bone volume, and bridge critical sized radial bone defects in adult mice, compared to implants without loaded BMP-2 (PCL + pPEA + FN), without FN (PCL + pPEA), or just PCL. Upper panel: X-ray images after surgery. Lower panel: 3D reconstructions from the $\mu C T$ images showing the radius in the area of the defect. Adapted with permission. ${ }^{[93]}$ Copyright 2018, Wiley. d) Coating of titanium implants with poly(ethyl acrylate) and FN allows osteoinduction of human mesenchymal stem cells (MSCs) at ultralow doses (ng $\mathrm{mL}^{-1}$ ) of BMP-7. Adapted with permission. ${ }^{[119]}$ Copyright 2018, American Chemical Society.

degradation and controlling their availability, local concentration, and bioactivity. ${ }^{[97]}$ It has hence found widespread use in developing biomaterial strategies for GF regulation or controlled release, for a variety of tissues and applications. For instance, in the case of BMP-2, it has been directly demonstrated that noncovalent surface immobilization through heparin augments its interaction with the cognate receptor while decreasing the interaction with its antagonist, Noggin.
This leads to higher levels of osteoinduction at lower doses than when BMP-2 is covalently linked or simply adsorbed to the supporting surface (see Figure 3a). ${ }^{[98]}$

Angiogenesis and neovascularization are essential for successful implantation of tissue-engineered constructs and cell transplantation, among other surgical procedures. It constitutes a central issue which focuses a great deal of attention, and several groups have begun to explore heparin for this purpose. 
The simplest way to incorporate heparin in a hydrogel system is to simply add it to the jellification mixture to trap it in the polymer network. This method avoids chemical reactions and shortens production processes. However, it leads to the release of heparin from the hydrogel over time, necessitating tuning procedures similar to the previously discussed controlled release systems (see Section 2).

This approach is desirable, anyhow, when the goal is to achieve a stable GF release, for example, to form soluble gradients for cell recruitment. Such a system based on type I collagen gels with incorporated heparin and pleiotrophin (PTN), a chemokine with recognized proangiogenic effects, has recently been tested. PTN release occurred at a nearly constant rate over 10 days, and selectively increased migration and viability of endothelial cells, compared to smooth muscle cells. As an additional advantage, heparin also increased the hemocompatibility of the gels, improving their eventual usefulness for endothelialization in vascular bypass grafts. ${ }^{\text {99] }}$

Alternatively, heparin can be covalently crosslinked into the gel network, leading to a more stable incorporation. For example, $N$-3-dimethylaminopropyl- $N$-ethylcarbodiimide (EDC) and NHS chemistry have been used to graft $N$-(3-aminopropyl) methacrylamide (NAPMAAm) onto heparin, to allow free radical polymerization along with PEG diacrylate (PEGDA). The resulting hydrogel was loaded with stromal derived factor- $1 \alpha(\mathrm{SDF}-1 \alpha)$, and was shown to stimulate chemotaxis of bone marrow cells in vitro. It could also selectively recruit anti-inflammatory (M2-like) macrophages in a spatially-localized manner in murine models. This effect was correlated with greater arteriolar remodeling in the injury niche, with sustained arteriogenesis and microvascular network growth throughout 7 days. These results demonstrate the potential of GF gradient-forming biomaterials for specific cell recruitment and neovascularization. ${ }^{100]}$

The ability of heparin to capture and present soluble factors can also be taken further beyond controlled release scenarios. The Healy group has performed a series of studies on HA/heparin combined hydrogels for presentation of TGF- $\beta 1$ and capture of endogenously produced angiogenic factors. The affinitybound TGF- $\beta 1$ induces an initial response on progenitor cells, which is then amplified by the sequestering of proangiogenic GFs produced endogenously in response to the initial stimulation. ${ }^{[101-103]}$ This provides a good example of the emerging trends of ECM-inspired microenvironment manipulation for control of stem/progenitor cell behavior. To prepare these gels, HA was modified with acrylate groups while heparin was thiolated, with thiol-ene chemistry being used to link the two polymeric components. An MMP-sensitive crosslinker was introduced into the system, allowing native cells to rearrange and degrade the scaffold throughout the regenerative process. These gels could stimulate the differentiation of both $\mathrm{Sca}-1^{+} / \mathrm{CD} 45^{-}$cardiac progenitor cells and human cardiosphere-derived cells into endothelial cells, and promote the in vivo formation of vascular-like structures which anastomosed with the host blood vessels. ${ }^{[101,103]}$ It was also discovered that these responses were superior when using highmolecular-weight heparin rather than low-molecular-weight or unfractionated heparin. ${ }^{[102]}$ These effects were the result of nitric oxide and VEGF production, which depended on the co-activity of CD105 (endoglin) with the TGF- $\beta$ R2 receptor. ${ }^{[103]}$
Feng et al. also developed an endogenous GF-sequestering hydrogel, by co-polymerizing two tyramine-modified polysaccharides, each with a specific function: Konjac glucomannan served as the building block and monocyte/macrophage activator, promoting secretion of proangiogenic GFs; heparin served its usual affinity-binding role. The designed hydrogels retained the properties of both components. Once subcutaneously implanted, they effectively sequestered the locally produced GFs in situ, promoting the formation and maturation of blood vessels in mice. ${ }^{[104]} \mathrm{HA} /$ heparin hydrogels functionalized with RGD-containing peptides by click chemistry have been tailored as a supporting scaffold for human pluripotent stem cell-derived neural progenitors. ${ }^{[84]}$ Neural stem/progenitor cell transplantation has been widely explored to promote brain tissue regeneration, for example, in Parkinson's disease patients. However, its (pre)clinical success has been limited by very low rates of cell survival during the procedure. ${ }^{[105]}$ Besides providing an adequate environment in terms of mechanical properties, these heparin-containing scaffolds were also able to sequester endogenous GFs. They could thus create a biologically amenable microenvironment, which led to nearly five times more dopaminergic neuron survival after transplantation into rat striatum. ${ }^{[84]}$

In a different application, SF has been combined with heparin to enhance GF activity at low doses by affinity binding and solid-phase presentation. ${ }^{[106]}$ Heparin was directly bound to SF by EDC/NHS chemistry and used to prepare bioactive biphasic SF scaffolds for tendon-to-bone interface (enthesis) engineering. These scaffolds integrated anisotropic (tendon/ ligament-like) and isotropic (bone/cartilage-like) pore alignment, and were able to present TGF- $\beta 2$ and growth/differentiation factor 5 (GDF-5) at low doses. Importantly, they could achieve biologically relevant effects on cultured ASCs. Results demonstrate that TGF- $\beta 2$ and pore anisotropy synergistically increased the expression of tendon/ligament markers, while combined presentation of TGF- $\beta 2$ and GDF- 5 enhanced expression of cartilage markers on areas with isotropic porosity. Interestingly, expression of enthesis markers was increased in the interface between the cartilage and tendon regions. ${ }^{[106]}$ This work shows how biomaterials designed for solid-phase presentation of select GFs can be leveraged to significantly improve and prolong their bioactivity vis-à-vis their soluble administration.

Alternatively, heparin can instead be conjugated to gelatin and then mixed with SF to form hydrogel biomaterials. ${ }^{[107]}$ As previously mentioned, SF lacks bioactive properties like cell adhesion motifs, which can be provided by combination with gelatin. Bragg et al. have studied the properties of such systems, concluding that sonication can accelerate the assembly process even in the presence of gelatin. Additionally, genipin crosslinking helps to stabilize both mechanical properties and biomolecule release from the hydrogels, achieving high retention of FGF-2 for at least 3 days. ${ }^{[107]}$

Another interesting application of heparin for GF presentation is the culture and harvest of cell sheets, particularly hepatocyte sheets. For example, poly( $N$-isopropylacrylamide-co2-carboxyisopropylacrylamide)-grafted tissue culture polystyrene surfaces were covalently grafted with heparin and used to affinity-immobilize EGF. Cultured rat primary hepatocytes 
could be maintained in culture without GF supplementation. Moreover, they had higher expression of hepatocyte-specific genes (albumin, hepatocyte nuclear factor $4 \alpha$, and coagulation factors VII and IX), and secreted higher levels of albumin than similar cells cultured on collagen-coated surfaces with EGF supplementation. Additionally, by simply lowering the temperature to $20^{\circ} \mathrm{C}$ after 4 day cultivation, it was possible to detach a cell sheet which retained fibronectin and EGF. ${ }^{108]}$

Another method of producing hepatocyte cell sheets was proposed by Hwang et al. ${ }^{[109]}$ A heparin-based hydrogel was micropatterned on a polyelectrolyte multilayer using photopolymerization and lithographic techniques. The system could precisely release a combination of HGF, EGF, and FGF, inducing ASC differentiation into the hepatic lineage. The cultured cells could then be recovered by electrochemical retrieval of cellattached micropatches. Upon intravenous injection, micropatches showed lengthy retention in the liver and promoted liver function in a rat model, when compared to the injection of hepatic-like cells cultured on tissue culture polystyrene. ${ }^{[109]}$

A variety of polymer matrices functionalized with heparin have been combined with different GFs and explored to promote osteogenesis. For example, heparin- $N$-(2-hydroxyl) propyl3 -trimethyl ammonium chitosan chloride nanocomplexes have been shown to load biologically relevant concentrations of BMP-2 and placental growth factor-2 (PlGF-2), while being able to stably retain over $80 \%$ for at least 21 days. Their combined delivery in in vitro assays resulted in enhanced osteoblast function compared to the delivery of each GF separately. ${ }^{[110]}$ In a different approach, decellularized bone matrix (DCB) has been used as an alternative biomaterial to enhance osteogenic differentiation of ASCs in vitro and in vivo. This material leverages the inorganic and organic components of the native bone ECM to maintain the majority of environmental cues of the original tissue. Recently, Rindone et al. have shown that the conjugation of heparin to DCB particles mimics the mechanism of GF tethering in a bone fracture healing microenvironment. The group used this technology to bind PDGF-BB, showing remarkable stability over time, with a release of $1.1 \%$ after 21 days, and over $90 \%$ encapsulation efficiency. The particles were then included in fibrin-PCL scaffolds and implanted in a murine calvarial defect model. These constructs significantly enhanced the deposition of $\mathrm{Ca}^{2+}$ and accelerated tissue mineralization, leading to more bone formation after 12 weeks. However, the expression of osteogenesis-related gene was the same in groups with and without PDGF-BB. A possible explanation is that the sequestering of endogenous GFs by heparin surpassed the effect of the administered PDGF-BB, and was sufficient to direct differentiation of implanted ASCs. ${ }^{[111]}$

Despite its usefulness, heparin has some associated limitations, such as lack of specificity due to molecular heterogeneity or its anticoagulant activity that may represent a risk in certain biomedical applications. ${ }^{[112,113]}$ To overcome some of the problems associated with the parent molecule, research groups have turned to molecules which mimic the effect of heparin, so-called heparin mimetics. These usually consist of highly sulfated polymer structures based on modified polysaccharides, synthetically sulfated oligosaccharides, oligosaccharide-aglycone conjugates, and noncarbohydrate-based sulfated mimetics. ${ }^{[113]}$ For example, an amphiphilic glycopeptide presenting trisulfated monosaccharides at the hydrophilic end was developed. ${ }^{[114]}$ These peptide amphiphiles could selfassemble into nanofibers and were shown to bind five different proteins without affecting their conformation. More impressively, it greatly amplified signaling of BMP-2 more than the natural heparin itself, promoting spinal bone regeneration with a 100 -fold lower dose than what is usually administered in soluble form to achieve the same effect. Another group followed a different strategy by modifying a methacrylated glycol chitosan hydrogel with sulfonated molecules, like polyvinylsulfonic acid or poly-4-styrenesulfonic acid (PSS). The modified gels preserved BMP-2 bioactivity after exposure to stressors such as acidic $\mathrm{pH}$, trypsin, or $37^{\circ} \mathrm{C}$ exposure for 7 days. Additionally, they also significantly enhanced osteogenic differentiation of encapsulated bone marrow stromal cells, with and without addition of exogenous BMP-2, with PSS gels achieving results remarkably similar to heparin-modified gels. ${ }^{[115]}$

Lastly, these GF-sequestering biomaterials based on heparin (or its mimetics) can be repurposed in a diametrically opposite way. Instead of being used to potentiate effects or control release rates, heparin can be used instead to scavenge and prevent the action of harmful or undesirable molecules, thereby modulating cell fate. A representative example of this approach is the use of star-shaped PEG hydrogels coupled with heparin derivatives for the capture of proinflammatory chemokines. ${ }^{[116]}$ This material could efficiently scavenge monocyte chemoattractant protein-1, interleukin-8, and macrophage inflammatory proteins $1 \alpha$ and $1 \beta$ in exudates from patients suffering from chronic venous leg ulcers. The authors showed how varying the desulfation pattern of heparin molecules led to different sequestering abilities for each chemokine, and estimated that the hydrogels are able to clean an entire pool of these cytokines at typical concentrations found in patients. Furthermore, this scavenging ability leads to a nearly $50 \%$ decrease in immune cell migration (both monocyte and polymorphonuclear cells) in in vitro Transwell assays, while also significantly decreasing immune cell infiltration in an in vivo model of delayed wound healing $(\mathrm{db} / \mathrm{db}$ mice). This contributed to a decrease in the overall inflammatory response and a faster regeneration of wounds. Increased re-epithelialization and more mature tissue were observed 10 days after application, even surpassing the effects of the commercial standard-of-care Promogran. ${ }^{[116]}$

A similar strategy was studied in a spinal cord injury setting, where a set of modified heparins desulfated at selected positions were tested for their ability to improve the regenerative process via sequestering of nefarious molecules. ${ }^{[17]}$ The authors observed that 2-O-desulfated/6-O-desulfated species enhanced neurite outgrowth and myelination, whereas the highly sulfated heparin control did not have verifiable positive effects. They further analyzed by mass spectroscopy the effects of these particular species on the protein contents of conditioned culture media. The authors identified several factors reported to play a role in damage or repair mechanisms, including amyloid $\beta \mathrm{A} 4$, which was confirmed to inhibit myelination in in vitro cultures. ${ }^{[117]}$ In another study, heparin/PEG microparticles were tested in an in vitro model of endochondral ossification. ${ }^{[118]}$ Results revealed that the particles bound insulin-like growth factor-binding proteins (IGFBP)-3 and -5, which led to a delayed process of differentiation similar to the 
addition of a small-molecule inhibitor of IGFBPs to the culture medium. ${ }^{[18]}$ These studies demonstrate the potential of heparin-based materials to inhibit undesired effects of certain molecules and modulation of cell microenvironments, ultimately producing significant effects on the fate of those cells.

Altogether, heparin-functionalized biomaterials have demonstrated the versatility of this molecule on the control of cell behavior and GF activity. However, the affinity binding between heparin and various GFs results from electrostatic interactions derived from sulfation patterns of the polysaccharide and the specific charge distribution of a given protein. As described over this subsection, a wide variety of molecules can bind with different affinities to heparin and other sulfated materials in a promiscuous manner. Although this can be leveraged for many purposes, achieving a fine control over specific biomolecule targets is challenging. Modulation of saccharide chain length/ molecular weight, fractionation, and sulfation patterns of specific groups has shown to generate some degree of selectivity in GFs sequestering. Nonetheless, these processes are still far from achieving an ideal solution. These limitations have propelled the search for alternatives to capture target GFs in a more specific manner, as will be discussed later.

Fibronectin-Based Platforms: Besides GAGs, there are other elements in the natural ECM with the ability to bind and retain GFs, which can be exploited for biomaterial development. Among them, FN has increasingly stood out for its multiple protein-binding motifs, which make it a multifunctional resource for the development of biomaterials. FN is a large glycoprotein widely present in connective tissues, consisting of two similar peptide chains linked by disulfide bonds to form $50 \mathrm{~nm}$ long arms. It contains several binding domains that link to a wide array of molecules-collagens, GAGs, other FN dimers, and integrins-highlighting its role as a "glue" holding tissues together. ${ }^{[20]}$ Interestingly, it has been progressively discovered that this protein contains several constitutive, as well as conformational-dependent, binding motifs for various GFs. ${ }^{[120-124]}$ Although the binding and activity regulation of TGF- $\beta 1$ and VEGF by FN have long been known, Martino and Hubbell have identified a specific highly promiscuous domain-12th to 14 th type III repeats, or FN III $_{12-14}$. FN III Fin-14 $_{14}$ is capable of interacting with the GFs of PDGF/VEGF and FGF families, and some GFs from the TGF- $\beta$ and neurotrophin families. ${ }^{[125]}$ This finding is particularly important when taking into account that the same protein contains a domain of high affinity for cell surface receptor integrins ( $\left.\mathrm{FN} \mathrm{III}_{9-10}\right)$. This means FN can allow synergistic activation of both types of receptors, a key advantage over heparin-based systems. ${ }^{[23]}$ Similar promiscuous binding domains were also discovered later in fibrin(ogen), which could open another avenue for the development of GF-sequestering biomaterials. ${ }^{[126]} \mathrm{FN}$ thus appears as a promising tool for bioactivity modulation via sequestering, which has led to two main types of strategies regarding its application.

One possibility is to design a biomaterial that can take advantage of the entire FN protein by rearranging its conformation so as to expose the desired cryptic domains. This has been the strategy explored in a series of works using poly(ethyl acrylate) (PEA) as a base polymer. ${ }^{[93,127-129]}$ Mechanistically, FN adsorption onto PEA promotes its spontaneous fibrillar reorganization, a process that has been termed "material-driven FN fibrillogenesis," while adsorption on other polymers results in retention of a globular conformation. This fibrillary conformation leads to a rearrangement of FN chains and simultaneous exposure of affinity-binding regions for both integrins and GFs. Remarkably, this behavior of FN on PEA surfaces is highly specific, even when compared to other closely related control polymers such as poly(methyl acrylate). ${ }^{\text {93,127-129] }}$

These FN conformational rearrangements have been shown to be crucial for enhancing GF presentation, attaining impressive results in vitro when used along with BMP-2 for driving osteogenesis of MSCs. Moreover, the same combination was able to drive full regeneration of a critical sized defect in vivo at concentrations over 300-fold lower than clinical standards. ${ }^{[127]}$ The ability of PEA to drive this favorable assembly has also been shown for 3D scaffolds, which greatly amplifies its applicability and translational potential. ${ }^{[128]}$ However, when FN was used to produce hydrogels, chemical crosslinking affected this process in a concentration-dependent manner, with reduction in segmental mobility being responsible for impairing the fibrillogenesis. Nonetheless, hydrogels with up to $2 \mathrm{wt} \%$ ethylene glycol dimethacrylate could still achieve the same results as those previously seen on 2D surfaces. ${ }^{[128]}$

It has also been demonstrated that plasma polymerized PEA, a production technique with more translational potential, can produce similar results. ${ }^{[93]}$ This approach was recently tested to coat polyimide tube implants, which provide a bioinert mechanical support for long bone fracture healing. In a mouse radial bone defect, it resulted in remarkable tomographical and histological results of newly formed tissue (see Figure 3c). ${ }^{[93]}$ Going a step further, this study used PEA-FN-BMP-2 coatings on decellularized bone chips inserted into the supporting tube implants for treatment of a chronic nonunion humeral fracture in an injured Münsterlander dog. While in this case the BMP-2 concentration was tenfold more than that used in the mouse model, it was still $10 \%$ of the dose typically used in veterinary applications for dog complex fractures. After conservative treatment had resoundingly failed to solve the problem, with amputation being seriously considered due to repeated Staphylococcus aureus infections, this tissue engineering approach resulted in full recovery of leg function without recurrence of infection 6 months after surgical intervention. ${ }^{[93]}$

Besides cooperative action with BMP-2, PEA-FN matrices have also been employed for VEGF binding to promote angiogenic ingrowth into tissue implants. ${ }^{[129]}$ Likewise, the synergistic effect of integrin/GF surface presentation proved to be important for improved VEGF action. Early onset of both signaling pathways was observed only in the fibrillary FN structures, while soluble VEGF or mutant FN with impaired integrin binding did not lead to similar effects. These effects were confirmed in vivo, with their implantation in murine fat pads enhancing formation of well-vascularized new tissue in the $3 \mathrm{D}$ scaffold pores. ${ }^{[129]}$

Recently, PEG-based hydrogels incorporating full-length FN have been investigated. ${ }^{[130]}$ The authors show that PEGylated FN still retains its cell-adhesive and fibrillogenic capabilities, and could be stably crosslinked into hydrogels with controllable stiffness and degradation rate. The covalent linking ensures that the protein remains stably incorporated in the gel network. Crosslinked hydrogels could still sequester VEGF and 
release it at stable rates over 3 days, with encapsulated endothelial cells forming multicellular and interconnected structures more extensively than in commercial Matrigel. In ovo and in vivo assays demonstrate the effect of incorporating VEGF and BMP-2 in these hydrogels to enhance angiogenesis in a chorioallantoic membrane assay and bone regeneration in mouse radial defect model, respectively. ${ }^{[130]}$

Using the entire FN protein, however, can result in high costs associated with its production and purification. Furthermore, the substrate upon which it is linked or adsorbed must be particularly optimized to enable the rearrangement of the native protein conformation, which could end up being a challenging and time-consuming empirical process. An alternative strategy to take advantage of the hitherto discovered binding domains is to use specific peptide sequences derived from those regions, in order to provide biomaterial scaffolds with GF- and integrin-binding capabilities. In theory, this is a less costly, more controllable, and reproducible alternative to the use of the full molecule. Several works have demonstrated the feasibility of this approach. ${ }^{[131,132]}$

HA hydrogels grafted with a structurally stabilized FN fragment containing the major integrin-binding domain $\mathrm{FN} \mathrm{III}_{9-10}$ have shown to greatly enhance the osteogenic effects of BMP-2 delivery. This was shown to happen mainly via improvement in MSC attachment and spreading, supported by integrin $\alpha_{5} \beta_{1}$ binding to the grafted sequences. In vivo, not only was the volume of ectopic bone formation in rat twice the one observed by delivering BMP-2 in unmodified HA hydrogels, but collagen fiber organization was also significantly improved..$^{[131]}$ Another study reinforced the role of synergistic integrin-GF receptor signaling mechanisms, and showed that the beneficial effect of FN in this regard could be emulated by engineering recombinant fragments to functionalize fibrin hydrogels. ${ }^{[132]}$ These polypeptide fragments contained a fibrin-binding sequence derived from factor XIII to allow their anchorage to the matrix, the integrin-binding domain $\mathrm{FN} \mathrm{III}_{9-10}$, and the GF-binding $\mathrm{FN} \mathrm{III}_{12-14}$. Interestingly, the authors also produced fragments containing only either FN III $_{9-10}$ or FN III $_{12-14}$. Results showed that even when both were present in the same matrix, the effect was not greater than their individual use. ${ }^{[132]}$ This emphasizes the fact that only when both domains are in close spatial proximity (in a single polypeptide chain), they synergistically lead to amplification of GF activity by promoting juxtacrine signaling mechanisms. Fibrin matrices grafted with this multifunctional peptide were examined for delivery of various GFs in different animal models, at GF concentrations which showed no significant effects when administered in soluble form. Particularly, when applied in a diabetic mouse model of chronic wound, formation of mature blood vessels could be stimulated by delivery of minimal doses of VEGF-A 165 and PDGF-BB (two to three orders of magnitude lower than those used in typical treatment regimens). Additionally, critical-sized calvarial defects underwent impressive healing after treatment with the multifunctional peptide-functionalized fibrin containing PDGF-BB and BMP-2, mostly through recruitment of stem/progenitor cells from the native surrounding tissues. ${ }^{[132]}$

Hay et al. have recently introduced an innovative approach to present these binding motifs on material surfaces. ${ }^{[133]}$ Lactococcus lactis bacteria able to spontaneously colonize a variety of material surfaces were engineered to become polypeptide-presenting units in order to control human MSCs in a highly dynamic temporal manner. L. lactis were engineered to express a membrane-bound $\mathrm{FN}_{\mathrm{III}} \mathrm{H}_{-10}$ (integrin-binding properties) creating a eukaryotic cell-biomaterial interface which allowed good cell adhesion and spreading. Furthermore, bacteria also expressed BMP-2 in four different ways: either constitutively or inducibly, in either membrane-bound or secreted form. Interestingly, secreted BMP-2 demonstrated pronounced osteogenic effects, unlike membrane-bound BMP-2. Moreover, in the inducible strains, BMP-2 expression could be triggered by addition of nisin, which activated the associated promotor. This allowed a temporal control over the production of the GF, and therefore triggered osteogenic differentiation in an ondemand manner. ${ }^{[133]}$

FN is indubitably a versatile and effective way to sequester and present GFs for tailored control of stem cell responses. Nonetheless, like heparin, the affinity-binding properties of its III $_{12-14}$ domain are promiscuous, which does not allow for the capture of a specific endogenous GF. The cellular response is therefore dependent on the addition of exogenous biomolecules and on attaining a precise control over their release behavior. The following section will expose different alternative strategies that have been developed to modify biomaterials in order to provide them with a GF-selective affinity that goes beyond what can be achieved with the previously described platforms.

\subsubsection{Selective Ligands for GF Capture}

In an effort to increase the precision of control over GF sequestration and, subsequently, cell responses, different affinity ligands with high selectivity for particular biomolecules have been increasingly researched. This subsection reviews the recent approaches on the use of antibodies, peptides, and aptamers as selective GF-binding partners for the development of intelligent biomaterials with molecular recognition properties (Table 2). Looking ahead in terms of evolution directions, a new generation of synthetic ligands with abiotic affinity produced by molecular imprinting technologies, which are beginning to make their way into the field, is also introduced.

Antibodies: Monoclonal antibodies (mAbs) constitute a model example of the aimed selective affinity function. Antibodies possess extremely high affinity $\left(K_{\mathrm{D}}\right.$ in nanomolar range) and specificity for their cognate target, being one of nature's most sophisticated recognition systems. ${ }^{[134]}$ Indeed, mAbs have become widely used therapeutics for a variety of diseases (e.g., oncology and autoimmune diseases) and assumed a major role in the field of targeted drug delivery. ${ }^{[135]}$ Some recent studies have also started to use them as tools to bind specific GFs. Several of these studies have focused on the functionalization of electrospun PCL nanofiber meshes for tissue engineering applications. ${ }^{[136-138]}$ A first proof-of-concept was developed using antibodies against TGF- $\beta 1$, FGF-2, and VEGF, either individually, with FGF-2 and VEGF antibodies being randomly mixed, or used side by side. ${ }^{[136]}$ All types of functionalization were shown to be successful in capturing their respective GF from a complex biological fluid, i.e., platelet lysate. Bioactivity of immobilized VEGF was shown to be retained, 
Table 2. Selective ligands for target GF capture and respective applications.

\begin{tabular}{|c|c|c|c|c|c|}
\hline Type & Ligand & Target molecule & Application & Support biomaterial & Refs. \\
\hline \multirow[t]{6}{*}{ Antibodies } & Mouse antihuman TGF- $\beta 1$ monoclonal antibody & TGF- $\beta 1$ & Proof of concept & $\begin{array}{l}\text { PCL electrospun } \\
\text { nanofiber meshes }\end{array}$ & [136] \\
\hline & Rabbit antihuman FGF-2 oligoclonal antibody & FGF-2 & & & \\
\hline & Mouse antihuman VEGF monoclonal antibody & VEGF & Vascularization & & \\
\hline & Mouse antihuman TGF- $\beta 3$ monoclonal antibody & TGF- $\beta 1$ & Chondrogenesis & & [137] \\
\hline & Goat antihuman IGF-I polyclonal antibody & IGF-I & & & \\
\hline & Mouse antihuman TNF- $\alpha$ monoclonal antibody & TNF- $\alpha$ & Anti-inflammatory action & & [138] \\
\hline \multirow[t]{12}{*}{ Peptides } & TSPHVPYGGGS & BMP-2 & Osteogenesis & $\begin{array}{l}\text { Peptide amphiphile } \\
\text { nanofiber gels }\end{array}$ & [143] \\
\hline & KGYPVHPST & & & PCL films/Polytape & [144] \\
\hline & KGLPLGNSH & TGF- $\beta 1$ & Tendon/ligament repair & PCL films & [145] \\
\hline & HSNGLPL & & Muscle regeneration & Polyurethane surfaces & {$[146,147]$} \\
\hline & & & Chondrogenesis & Chitosan sponges & [148] \\
\hline & $(\mathrm{DDDDS})_{5}$ & FGF-2 & Proof of concept & $\begin{array}{l}\text { Artificial matrix } \\
\text { composed } \\
\text { of } 12 \text { repeats } \\
\text { of APGVGV }\end{array}$ & [149] \\
\hline & KRTGQYKL & Heparin & $\begin{array}{c}\text { Amplification } \\
\text { of supplemented GF effect }\end{array}$ & $\begin{array}{l}\text { Oligo(ethylene glycol) } \\
\text { (OEG) self-assembled } \\
\text { monolayers (SAMs) }\end{array}$ & [150] \\
\hline & $\begin{array}{l}\text { Laminin peptide } \alpha_{2} \mathrm{PI}_{1-8} \text {-LAMA3 }{ }_{3043-3067} \\
\text { (RLVFALGTDGKKLRIKSKEKCNDGK) }\end{array}$ & VEGF-A165 and PDGF-BB & Skin wound healing & Fibrin matrices & [151] \\
\hline & Kcoil $(\text { KVSALKE })_{5}$ & $\begin{array}{l}\text { Ecoil }(\text { EVSALEK) })_{5} \text {-tagged } \\
\text { recombinant EGF and VEGF }\end{array}$ & Vascularization & Chondroitin sulfate surfaces & [152] \\
\hline & & $\begin{array}{l}\text { Ecoil-tagged recombinant } \\
\text { EGF and FGF-2 }\end{array}$ & Proof of concept & Gelatin hydrogels & [153] \\
\hline & $G \ln -Z Z$ & $\begin{array}{l}\text { Fc-tagged recombinant } \\
\text { PDGF-BB }\end{array}$ & $\begin{array}{l}\text { In vitro modeling } \\
\text { of perivascular niche }\end{array}$ & PEG hydrogels & [154] \\
\hline & $\begin{array}{c}\operatorname{KE}\left\{F_{d}\right\}\left\{A_{d}\right\}\left\{Y_{d}\right\}\left\{L_{d}\right\} \\
\text { IDFNWEYPASKCKSAPYEWNFDI }\left\{L_{d}\right\}\left\{Y_{d}\right\}\left\{A_{d}\right\}\left\{F_{d}\right\} \\
\text { EK }\end{array}$ & VEGF & Inhibition & PEG microspheres & [155] \\
\hline \multirow[t]{12}{*}{ Aptamers } & $\begin{array}{c}\text { /ThiolMC6-D/AAAAA AAAAA CCCGT CTTCC } \\
\text { AGACA AGAGT GCACG C }\end{array}$ & VEGF & Skin wound healing & Fibrin hydrogels & {$[156,157]$} \\
\hline & $\begin{array}{l}\text { GAGGA CGAUG CGGAA UCAGU } \\
\text { GAAUG CUUAU ACAUC CG invT }\end{array}$ & & Vascularization & PEG hydrogels & [158] \\
\hline & & & & $\begin{array}{l}\text { PEG-gelatin } \\
\text { hydrogels }\end{array}$ & [159] \\
\hline & СCCGT CTTCC AGACC AAGAG TGAGG G & & & PEG hydrogels & [160] \\
\hline & СCCGT СTTCC AGACA AGAGT CGAGG G & & & Chitosan hydrogel films & [161] \\
\hline & $\begin{array}{c}\text { AGGGC CACGT CTATT TAGAC TAGAG TGCAC } \\
\text { TGGTT C }\end{array}$ & & & $\begin{array}{l}\text { Polyacrylamide gels; } \\
\text { collagen sponges }\end{array}$ & [162] \\
\hline & $\begin{array}{c}\text { GCGGG GCTAT GTAAA TTACT } \\
\text { GCTGT ACTAC GC }\end{array}$ & FGF-2 & & PEG hydrogels & [160] \\
\hline & $\begin{array}{l}\text { /5ThioMC6-D/GCGAT ACTCC ACAGG CTACG } \\
\text { GCACG TACAC CATCA CCATG ATCCC A }\end{array}$ & PDGF-BB & & Fibrin hydrogels & [157] \\
\hline & $\begin{array}{l}\text { AAAAA AAAAA ACACG CTACG GCACG TACAG } \\
\text { CATCA CCATC ATCCT GTGAC TTGAC C }\end{array}$ & & & Polystyrene microparticles & [163] \\
\hline & $\begin{array}{l}\text { GCGAT ACTCC ACACG CTACG GCACG TACAG } \\
\text { CATCA CCATC ATCCT C }\end{array}$ & & & & [164] \\
\hline & $\begin{array}{c}\text { ACAGG CTACG GCACG } \\
\text { TAGAG CATCA CCATC ATCCT G }\end{array}$ & & & & [163] \\
\hline & & & & Chitosan hydrogel films & [161] \\
\hline
\end{tabular}


Table 2. Continued.

\begin{tabular}{|c|c|c|c|c|c|}
\hline Type & Ligand & Target molecule & Application & Support biomaterial & Refs. \\
\hline & $\begin{array}{c}\text { CAGGC TACGG CACGT AGAGC } \\
\text { ATCAC CATCA TCCTC }\end{array}$ & & & $\begin{array}{l}\text { Polyacrylamide gels; } \\
\text { collagen sponges }\end{array}$ & [162] \\
\hline & $\begin{array}{l}\text { NH2-[A]9-AGGTC AGATG AGGAG GGGGA } \\
\text { CTTAG GACTG GGTTT ATCAC CTATG CGTG }\end{array}$ & $\begin{array}{c}\text { Alkaline phosphatase } \\
\text { (and other epitopes) } \\
\text { on pluripotent } \\
\text { stem cell surfaces }\end{array}$ & MSC recruitment & $\begin{array}{l}\text { SF sponge }+ \text { SF/HA-tyramine } \\
\text { hydrogel }\end{array}$ & [165] \\
\hline & $\begin{array}{l}\text { GGGG TGGGT GGGGG GCACG } \\
\text { TGTGG GGGCG GCCAG GGTGC T }\end{array}$ & Fractalkine & $\begin{array}{c}\text { Modulation of inflammation } \\
\text { via nonclassical monocyte } \\
\text { recruitment }\end{array}$ & PEG hydrogels & [166] \\
\hline
\end{tabular}

since endothelial cell cultures on functionalized substrates had increased DNA, total protein, and intracellular VEGF contents. ${ }^{[136]}$

This concept has been further explored in the development of bioactive scaffolds to promote chondrogenic differentiation ${ }^{[137]}$ and to modulate the action of proinflammatory cytokines. ${ }^{[138]}$ In the first case, anti-TGF- $\beta 3$ and anti-insulin-like growth factorI (IGF-I) antibodies were tethered to the fiber scaffolds, and tested for the effects on human bone marrow-derived MSCs cultured under basal culture medium over 28 days. Observation of spherical morphology typical of chondrocytes and deposition of collagen type II suggested the formation of a cartilage-like ECM (Figure 4a). ${ }^{[137]}$ In the second case, the goal was to use an antibody which inhibited the binding of the well-known proinflammatory cytokine tumor necrosis factor- $\alpha$ (TNF- $\alpha$ ) to its receptor, in order to decrease its deleterious effects in articular diseases such as rheumatoid arthritis. Antibody-grafted scaffolds could capture and retain TNF- $\alpha$ produced by macrophages, while primary human articular chondrocytes could be cultured on the meshes for 28 days with retention of their typical phenotype. ${ }^{[138]}$ However, it remains to be assessed if the ability to capture the cytokine can provide benefits to the cultured chondrocytes.

Nevertheless, some important limitations have stifled the expansion of the use of antibodies in this context, particularly potential immunogenicity due to their mammalian origin, stability issues, and lack of cost-effective production methods. ${ }^{[139]}$ In sum, many of the problems assigned to the application of exogenous recombinant human GFs can also be pointed for $\mathrm{mAbs}$, indicating the need of smaller, more stable alternatives to develop this strategy. Recently, novel molecules based on the function of antibodies (particularly single-chain variable fragments) have begun to be engineered in order to retain the original avidity while improving pharmacokinetic properties. ${ }^{140-142]}$ These developments may open another avenue of research, but so far the majority of the work on the subject has been dedicated to two other types of molecules: peptides and aptamers.

Peptides: The use of peptides as targeted ligands for specific proteins is not new and has become increasingly relevant across various areas of research, such as in separation and purification processes or targeted drug delivery, for example. ${ }^{[167,168]}$ A number of advantages make these small molecules attractive for affinity binding of specific targets. The relatively large collection of monomers that can be used for their synthesis leads to a nearly infinite number of combinations with different properties, especially if one includes noncanonical amino acids into the mix. This allows the creation of chains with high specificity and affinity, which can be discovered via high-throughput screening methods, particularly phage display technologies. ${ }^{[169]}$ Briefly, this consists on the display of random peptide sequences by bacteriophages, which then undergo a cycle of incubation with the target molecule, affinity purification by washing of low-affinity unbound phages, followed by recovery and expansion of the specifically bound fractions. This selection process is called biopanning, and usually three to five rounds are performed in order to obtain targets that bind with high affinity. ${ }^{[169]}$ Furthermore, peptides can be easily modified with a particular amino acid to allow coupling by a predetermined chemical reaction, with thiol-containing cysteine, imidazole-containing histidine, or amine-containing lysine being the most common.

It is not surprising then that peptides have attracted particular attention for targeting GFs by affinity binding. They have been tested, for example, to sequester BMP-2 in order to stimulate bone regeneration. ${ }^{[144]}$ In one study, Crispim et al. tethered KGYPVHPST peptides, which had previously shown affinity for BMP-2, to PCL films via EDC/NHS coupling. ${ }^{[144]}$ The modified films were shown to capture soluble rhBMP-2, and $\mathrm{C} 2 \mathrm{C} 12$ Luc reporter cells confirmed the bioactivity of the immobilized GF. The peptide was further tested in vivo, being used to functionalize commercially available Polytape, which was sutured together with a graft used for anterior cruciate ligament reconstruction in rats. Without addition of any exogenous GF, peptide-functionalized Polytape was able to enhance bone formation inside femoral and tibial tunnels by capturing endogenous BMP-2, and ultimately improve the graft quality. ${ }^{[144]}$ This strategy was also followed for the capture of TGF- $\beta 1$ to promote tendon regeneration. In vitro, target genes on human primary tendon cells were activated by the immobilized rhTGF- $\beta 1$, with collagen production increasing 2.5 -fold. In vivo implantation in rats not only showed accumulated TGF- $\beta 1$ on the functionalized film surface, but also increased recruitment of immune cells, fibrogenic response, and vascularization around the implant. These results revealed that the bound GF maintained its bioactivity and contributed to modulating the local response to the implant. ${ }^{[145]}$

TGF- $\beta 1$ has also been targeted in other studies for muscle and cartilage healing, with similar degrees of success. Xiao et al. coupled the affinitive peptide HSNGLPL to a polyurethane (PU) substrate via copper-catalyzed azide-alkyne cycloaddition 

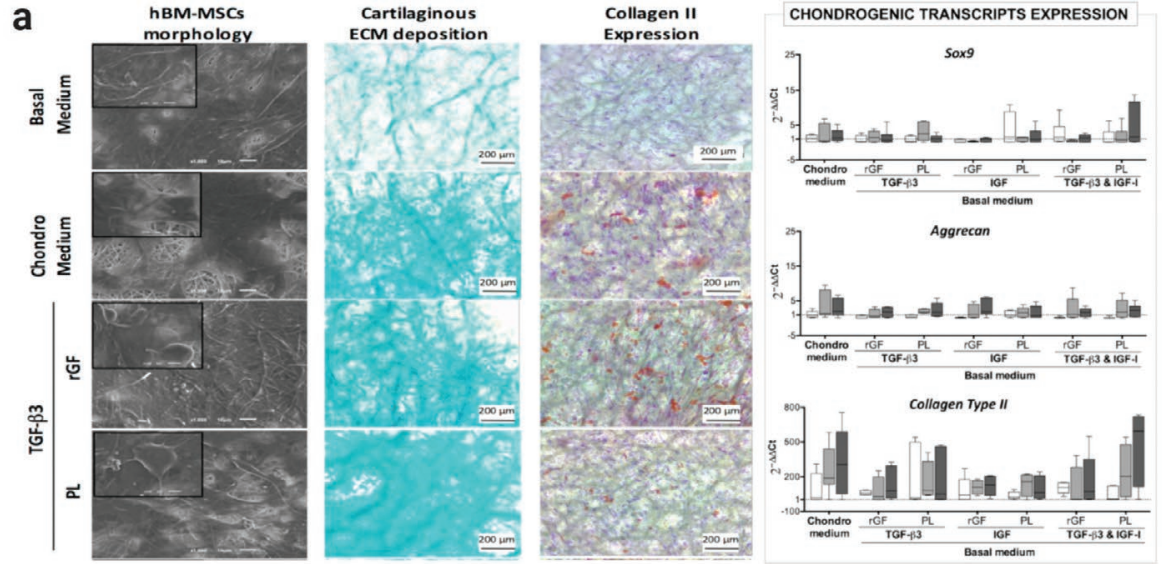

b

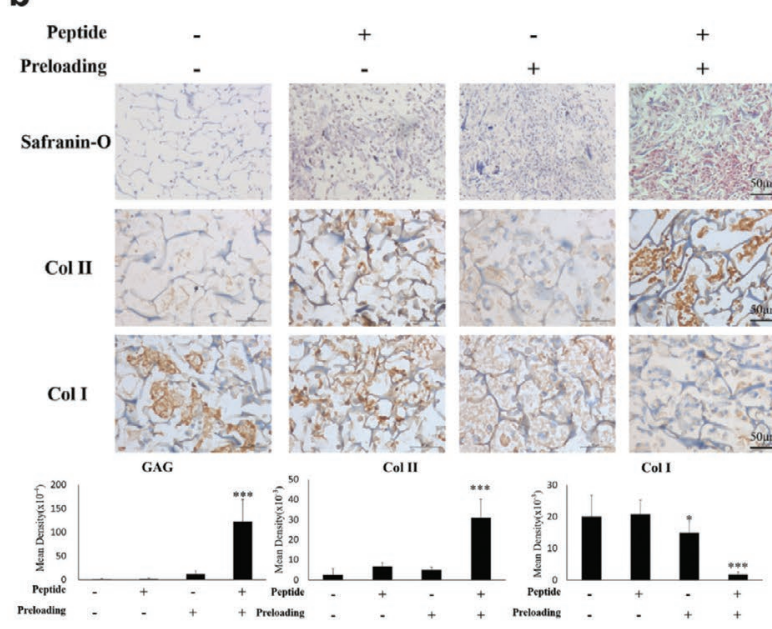

C

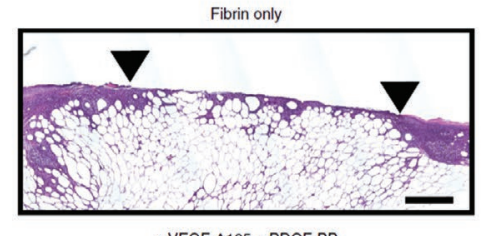

+ VEGF-A165 + PDGF-BB

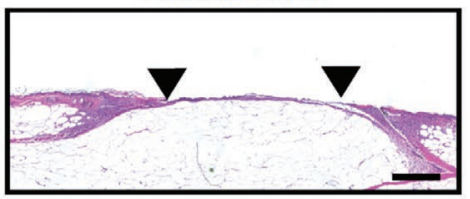

$+\alpha 2$ PI1-8-LAMA3 3 2043-0e7 + VEGF-A165 + PDGF-BB

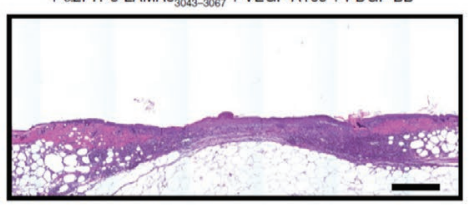

d
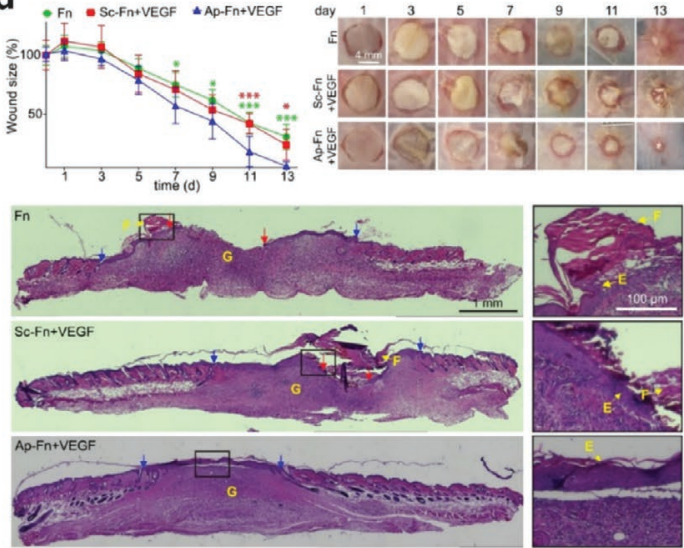

e
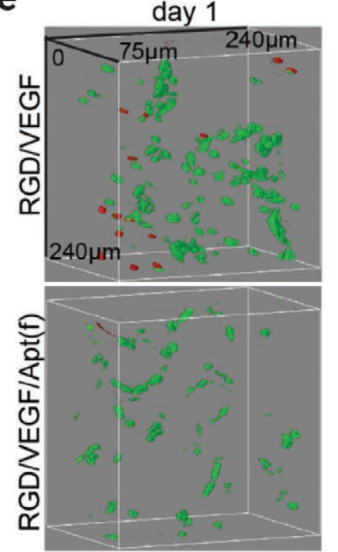

day 7
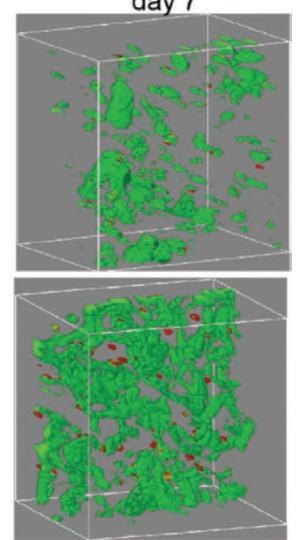

Figure 4. Biomaterial functionalization with selective ligands for GF capture. a) Anti-TGF- $\beta 3$ antibody-grafted electrospun PCL nanofiber meshes induced chondrogenic differentiation of MSCs similarly to standard chondrogenic differentiation medium. Left: ECM deposition (collagen type II and GAGs). Right: expression of chondrogenic markers. Adapted with permission. ${ }^{[137]}$ Copyright 2019, Elsevier. b) TGF- $\beta 1$ affinity peptides incorporated within a chitosan-sponge scaffold significantly enhance cartilage regeneration. Preloading of peptide-functionalized scaffolds with encapsulated MSCs resulted in higher deposition of cartilaginous matrix. Adapted with permission. ${ }^{148]}$ Copyright 2018, The Royal Society of Chemistry. c) Fibrin gels functionalized with laminin peptide LAMA $3_{3043-3067}$, combined with the delivery of VEGF-A ${ }_{165}$ and PDGF-BB, enhanced skin wound healing of full-thickness back-skin wound in mice, compared to fibrin only or to formulations with GFs but without the laminin peptide. Adapted under the terms and conditions of the Creative Commons Attribution 4.0 International License. ${ }^{[151]}$ Copyright 2018, The Authors, published by Springer Nature. d) Aptamer-functionalized fibrin hydrogels delivering VEGF significantly hastened skin wound closure and re-epithelialization in mice. Adapted with permission. ${ }^{[156]}$ Copyright 2019, American Chemical Society. e) Functionalization of VEGF-loaded 3D porous PEG hydrogels with a VEGF-specific aptamer promotes HUVEC growth in comparison with gels with no aptamer. Adapted with permission. ${ }^{[158]}$ Copyright 2017, Wiley. 
(CuAAC) click reaction, and implanted fragments of the material into mice gastrocnemius muscles. ${ }^{[146]}$ Peptide-grafted fragments with previously adsorbed TGF- $\beta 1$ greatly sped up the ensuing immune response and tissue regeneration. Increased collagen production was observed throughout the experimental period, and newly formed muscle fibers replaced the necrotic area surrounding the implants by day 21 . The authors also analyzed the adsorption of TGF- $\beta 1$ using quartz crystal microbalance with dissipation. Results showed that adsorption to the peptide-modified PU surfaces occurs through the formation of a loose and diffuse layer, indicating stable spatial conformation, whereas on native PU surfaces it formed a compact and rigid layer. These results provide a possible physical justification for the superior GF bioactivity when bound to its affinitive peptide. ${ }^{[146]}$ However, in this study, exogenous GF was added to the scaffolds, and the authors do not make any comparison between the administered dose and the ones typically used with soluble administration. The same group has further shown that implantation of peptide-grafted scaffold fragments without preadsorption of the GF also resulted in enrichment of TGF- $\beta 1$ at the implant site (likely produced by infiltrating macrophages). This led to a reduction in necrosis and a faster regenerative response, contrasting with unmodified PU fragments, around which no formation of new muscle fibers could be observed. ${ }^{[147]}$ The same peptide was tested with porous chitosan scaffolds for enhancing cartilage regeneration. ${ }^{[148]}$ Upregulation of chondrogenic-related genes such as Sox9, Col II, and AGG in cultured MSC was positively correlated with the amount of TGF- $\beta 1$-binding peptide. MSC-colonized scaffolds with preloaded TGF- $\beta 1$ implanted in nude mice models resulted in significant formation of ectopic cartilage (Figure $4 \mathrm{~b}$ ), while in an osteochondral defect model, cartilage regeneration was significantly promoted even in the absence of exogenous factors and seeded cells. ${ }^{[148]}$

Another innovative use of affinity peptides was presented by Lee et al., who developed peptide amphiphiles which could self-assemble into nanofibers. ${ }^{[143]}$ These not only presented a BMP-2 affinity-binding sequence at their hydrophilic surface but could also be used to build a gel scaffold with osteoinductive properties. These scaffolds were tested for arthrodesis in a rat posterolateral lumbar intertransverse spinal fusion model, achieving superior fusion rates with a tenfold reduced dose of BMP-2. Moreover, a $42 \%$ fusion rate was observed without the use of any exogenous GF, demonstrating that the peptide can specifically and effectively sequester the native factor from the surrounding environment in vivo. ${ }^{[143]}$ A different peptidebased strategy was followed by Suttinont et al. In this work, the authors designed an artificial ECM made of 12 repeats of the APGVGV peptide motif derived from elastin as the structural basis, the commonly used RGD motif to promote cell adhesion, and an acidic amino acid-rich sequence composed of five repeats of four aspartic acids and a serine (DDDDS). This sequence was chosen in order to electrostatically interact with FGF-2, since this GF has a highly basic domain, thus allowing affinity immobilization. MSC-like C3H10T1/2 cells cultured on the engineered matrices had increased proliferation without the addition of exogenous FGF-2, demonstrating that scaffolds were able to capture the GF produced by the cells and maintain its signaling. ${ }^{[149]}$ On the downside, the specificity of the peptide used in this study was not of the same order of peptides discovered through phage display, being more similar to previously described strategies that made use of heparin for electrostatic interactions. Further experiments with complex medium formulations should elucidate the ability of this type of scaffolds to distinguish between different proteins.

Another perspective on the use of peptides as affinity ligands was presented by Hudalla et al. The group was inspired by the interaction between FGF-2 and heparin, and instead of seeking a peptide to bind the GF, designed a sequence derived from FGF-2 to bind and capture serum-borne heparin..$^{[150]}$ In turn, the sequestered heparin would be able to bind and present soluble FGF-2. Thus, the electrostatic interactions between the two molecules could be advantageously explored for their alternate capture from biological fluids, avoiding the use of both exogenous heparin and GF. To prove this point, oligo(ethylene glycol) (OEG) self-assembled monolayers (SAMs) were chosen to diminish nonspecific protein adsorption. SAMs were modified with the FGF-2-derived peptide KRTGQYKL, along with the usual RGD peptides. HUVECs cultured on the substrates responded to FGF-2 supplementation with increased proliferation but only if fetal bovine serum, which contains heparin, was also present, meaning that the surface could not sequester the GF directly. ${ }^{[150]}$ Although this approach is interesting for its use of both heparin- and peptide-based affinity scaffolds, the promiscuity of heparin is still an issue. Targeting of FGF-2 could only occur because this particular factor was supplemented into the medium, which does not completely solve the problems related to exogenous administration.

Other peptides that bind GFs can be found in the heparinbinding domains of laminin. Laminin is an abundant ECM glycoprotein, with especially important functions in attaching cells to the matrix, being one of the main proteins present in the basal lamina of epithelia. ${ }^{[20]}$ Similar to previously discussed FN, laminin contains particular domains, which are able to bind GFs in order to regulate their activity in the native milieu. Ishihara et al. used peptidic sequences derived from this protein to engineer GF-sequestering biomaterials. ${ }^{[151]}$ By covalently incorporating LAMA3 L $_{3043-3067}$ and LAMA5 ${ }_{3417-3436}$ peptides engineered to contain a TG-sensitive domain, fibrin matrices could stably retain VEGF-A 165 and PDGF-BB, both in vitro and in vivo in diabetic wound models. Co-delivery from the fibrin matrix onto full-thickness back-skin wounds in $\mathrm{db} / \mathrm{db}$ mice, which provides a well-established model of impaired wound healing, resulted in significant speeding up of the regenerative process (Figure 4c). This strategy not only promoted a faster wound closure, as it led to the formation of more granulation tissue, and had a positive effect on the neutrophil/monocyte balance. Unfortunately, the authors did not observe significant effects when the peptide-grafted matrices were implanted without preloading of GFs. But interestingly, it performed better in terms of wound closure than fibrin matrices containing the two GFs without the peptides. ${ }^{[151]}$ This supports the concept that strategies focused on sequestering endogenous molecules may indeed be a more advantageous alternative to the exogenous administration of recombinant proteins. Nevertheless, like FN, this group of peptides also presents the problem of binding promiscuity. Though they might bind the desired GFs if adsorbed prior to implantation, when placed in complex 
biological environments, they are incapable of distinguishing between the target GFs and others with similar affinity. Therefore, they do not allow a precise directed control over cellular responses. To a certain extent, this explains why the matrices without loaded factors did not achieve a significant effect upon implantation in vivo.

A different concept to leverage on peptide affinities is the formation of coiled coil structures between two peptides with complementary coil domains. ${ }^{[152-154]}$ This strategy implies modifying the target GF with one of the peptide tags (usually by recombinant technology), while immobilizing the other one at the biomaterial surface. Then, by incubating both together, the coil peptides will link with one another, functionalizing the biomaterial with the factor(s). Furthermore, correct tagging of the GF will allow maintenance of its bioactivity, since the surface immobilization occurs via noncovalent binding and does not affect the protein conformation nor the receptor-binding region. ${ }^{[152-154]}$

This concept has been explored for several GFs and different regenerative applications. For example, tagged EGF and VEGF were both tethered to a chondroitin sulfate surface functionalized with complementary coil peptides, resulting in major increases in endothelial and smooth muscle cell survival after 2 days in serum-free medium. ${ }^{[152]}$ Another study designed and produced a novel linker protein, containing a coil peptide domain to link with tagged GFs on one hand, and a collagenbinding domain that binds gelatin on the other. Using this protein, the authors captured FGF-2 and EGF modified with the complementary coil peptide to the surface of gelatin scaffolds. This method preserved GF bioactivity, increasing smooth muscle cell survival and endothelial cell proliferation in vitro in reduced serum and serum-free conditions. ${ }^{[153]}$ In a different application of this concept, PDGF-BB was combined with a PEG-based scaffold to construct an in vitro model of the perivascular niche. In order to study the behavior of MSCs resident in this microenvironment, the authors constructed an engineered niche, analogous to its in vivo counterpart. This consisted of an artificial perfusable vessel, a proteolytically degradable matrix with integrin-binding sites, and a stable gradient of immobilized PDGF-BB, decreasing from the vessel area outward. When PDGF-BB is administered by typical soluble delivery, MSCs respond by proliferating, spreading, and migrating. In contrast, matrix-tethered PDGF-BB gradients resulted in locally restricted morphogenetic responses, similar to what would be expected in a native perivascular niche. ${ }^{[154]}$ Despite the benefits of this approach, it necessitates the production of recombinant proteins with a specific binding domain in order to tether them to the peptide-grafted matrix. This introduces an element of complexity and increases production costs, restricting the benefits of using affinity-binding peptides.

Lastly, nano- or microparticles functionalized with targeted peptides can also be applied to mimic the behavior of natural inhibitors that bind the soluble GFs and prevent their interactions with the cognate receptor. This inhibitory effect is usually achieved by interfering with the receptor-binding region, effectively hiding it from cells. As an example, Belair et al. dampened the activity of VEGF derived from human platelets using PEG microspheres containing a VEGF-binding peptide derived from VEGFR2. ${ }^{155]}$ When applied to HUVECs cultured with platelet concentrate, proliferation was manifestly reduced, due to the sequestration of VEGF by the microspheres, which decreased the concentration of the GF that could interact with cell receptors. The use of divalent peptides greatly increased this effect, showing that it is possible to tune the behavior of these materials using different ligands. ${ }^{[155]}$

Despite the continued improvements and the multiple ways in which peptides can be used as affinity linkers for GFs, some limitations still remain. First, discovery of peptide ligands for specific GFs and their synthesis can prove to be a costly process, especially if they require a specific conformation to exert proper effect. Furthermore, their conjugation to biomaterials with site specificity can be challenging for those without synthetic chemistry expertise or use of noncanonical amino acids. Another identified issue is that the narrow number of sequences identified from phage display does not necessarily lead to the most promising binding motifs, due to identification of false positives. This can occur due to different phage propagation rates, for example. Moreover, this process can be slow and laborious, missing out on promising sequences due to methodological limitations. ${ }^{[170]}$ Lastly, susceptibility to proteolytic degradation is also a concern, which can limit the stability of peptides in vivo and thereby reduce their effectiveness. ${ }^{[168]}$

Aptamers: Aptamers have been increasingly researched as an alternative for applications typically associated with antibodies and peptide ligands, such as targeted therapeutics and diagnostics, peptide, and protein inhibition, ${ }^{[171]}$ and more recently GF sequestration. Aptamers are single-stranded oligonucleotides selected from synthetic RNA/DNA libraries, which bind to target molecules with both high affinity and specificity, being able to differentiate targets by subtle structural differences. ${ }^{[157,160]}$ Moreover, when compared to larger molecules (e.g., antibodies and proteins), they present a number of advantages that make them attractive molecules for selective recognition purposes. Aptamers are considerably easier and cheaper to prepare and scale up since they usually consist of short sequences (of 20-60 nucleotides) that can be synthesized and modified with standard chemical synthesis and conjugation methods. Additionally, modifications for the attachment of dyes or functional groups are orthogonal and can be readily introduced during synthesis, greatly increasing the versatility of these platforms. Other positive aspects usually associated with their nucleotide nature are low immunogenicity or toxicity potential; the fact that they are gradually degraded by nucleases and cleared from the organism; and the possibility for synthesis manipulation to tune their stability in biological environments. ${ }^{[157,160,165,170]}$ Lastly, aptamers possess a unique ability related to principles of nucleic acid double-strand formation: they can be quickly released from their targets by the effects of complementary strand displacement, which can be understood as an "on/off trigger" mechanism. By contrast, peptides, for instance, are inherently monomodal, constantly being switched "on" at all times. This faculty confers to aptamerfunctionalized systems a further level of temporal control that is essential to restrict biological responses to their relevant time window. ${ }^{[157,160,165,170]}$

An increasing number of works have demonstrated the utility of aptamers for sequestering soluble proteins such as GFs. The group of Wang, in particular, has developed several systems for 
stable retention and complementary strand-triggered release of the captured protein. ${ }^{[156-160,163,164]}$ PEG diacrylate gels have been used as base materials for most of these works, serving as controllable synthetic hydrogel platforms which can be readily modified with the aptamers. The authors began by developing a superporous material using free radical polymerization coupled with gas foaming, with the goal of attaining high uptake and loading of target molecules, as well as allowing space for cell migration and transport of nutrients and oxygen. This hydrogel system incorporated a modified form of a previously developed PDGF-BB-specific aptamer, which was shown to sequester over $98 \%$ of the free GF in solution $\left(0.3 \mathrm{ng} \mathrm{mL}^{-1}\right)$ in a microparticle assay. Functionalized hydrogels could retain over $90 \%$ of sequestered PDGF-BB for at least $24 \mathrm{~h}$, at various concentrations, as long as the aptamer/GF ratio was at least 10:1. Moreover, other aptamers with different affinities were also developed in order to tailor the retention kinetics, and complementary sequences could also be added to prompt a short-term burst release of the captured protein, making this a very flexible and controllable system. ${ }^{[164]}$

Subsequent work has demonstrated that this scaffold can be further combined with more aptamers, peptides, or other molecules, increasing their complexity and functionality. For example, a double aptamer modification was performed to stimulate higher degrees of angiogenesis by capturing both VEGF and FGF-2. The rationale behind this strategy is that the use of a single GF only leads to transient effects, which could be responsible for the poor outcomes of clinical trials using VEGF.[172,173] Both in vitro endothelial cell migration and in ovo blood vessel formation and maturation were notably increased by using these dual hydrogels. ${ }^{[160]}$ This approach was taken a step forward by patterning an allyl chitosan hydrogel. Photocoupling and thiol-ene chemistry were combined to produce a grid-like pattern of aptamers on the gel surface. This resulted in an ability to uptake 4-5 times more GFs than unmodified hydrogels, which relied simply on diffusion mechanisms. The films were successfully applied to complex matrices such as human serum, and demonstrated precisely controlled spatiotemporal release and excellent biocompatibility. Yet, functional assays were not performed on relevant cell types, to determine the ability of the scaffold to protect the bioactivity of the captured GFs and exert the desired effects. Nor were the target factors sequestered from the complex matrices, which would have been a more relevant result from an application standpoint, considering the aimed reduction of exogenous GF use. ${ }^{[161]}$

Another example of a system with dual modification is the addition of an anti-integrin RGD-containing peptide along with an anti-VEGF aptamer to a PEG hydrogel. This significantly increased cell attachment in a concentration-dependent manner, which was essential in the generation of an angiogenic response by seeded endothelial cells (Figure 4e). ${ }^{[158]}$ A chimeric version of this hydrogel has also been studied. In order to further mimic the ECM and increase its biofunctionality, PEG was combined with gelatin and the anti-VEGF aptamer. Sequestered VEGF maintained $\approx 50 \%$ bioactivity during a 14 day test, while gelatin contributed to cell adhesion and colonization of the scaffold. This system helped HUVECs survive the duration of the test in serum-reduced culture conditions and establish some intercellular connections. ${ }^{[159]}$ It should be noted, though, that the aptamer used in this study is a commercial inhibitor of VEGF, used to treat age-related macular degeneration. ${ }^{[174]}$ This means that the GF was sequestered in an inactive form, and only acted upon the cells when it was released over time. ${ }^{[159]}$ That could indicate that the strategy followed here did not take full advantage of the abilities of the modified scaffold, and may potentially be improved by the development of new binding molecules that retain or even amplify the activity of VEGF, instead of decreasing it.

Recent studies have coupled the use of these aptamers with fibrinogen in order to create in situ gelling GF-sequestering scaffolds. As previously mentioned, in the blood coagulation cascade, fibrinogen is activated by thrombin to assemble into fibrin fibrils that are crosslinked by FXIIIa into networks that sustain clots. Fibrin matrices have been explored as natural scaffolds for tissue engineering purposes, and this characteristic makes this material interesting for injectable applications. ${ }^{[175,176]}$ Zhao et al. thus produced bifunctional aptamerfibrinogen macromers, which possessed a dual capability of molecular recognition and self-assembly. These macromers were successfully polymerized into VEGF- and PDGF-BBsequestering fibrin matrices by thrombin activation, being able to sustain GFs' bioactivity and release over 15 days. In vitro assays show remarkable angiogenic responses from seeded endothelial cells, as well as sprouting from cultured aorta ring sections. Moreover, subcutaneous injection into mice promoted the growth of mature blood vessels after 20 days ${ }^{[157]}$ and significantly improved wound closure after 13 days. ${ }^{[156]}$

Stejskalová et al. have recently presented an elegant strategy to control the sequestering and release of specific GFs. ${ }^{[162]}$ The authors developed a programmable nanotechnology-based platform that bypasses the use of traditional triggers for the release of bound proteins, such as exogenous inputs (e.g., light), spatially diffuse triggers (e.g., $\mathrm{pH}$ shifts), or passive activation (e.g., hydrolysis). After its natural secretion, TGF- $\beta 1$ is sequestered by the large latent complex (LLC), which maintains it inactive in the ECM until a mechanical stimulus triggers its release. ${ }^{[177]}$ Inspired by the LLC, the group developed a flexible aptamer technology that harnesses cellular traction forces to activate GF release. This concept was shown to work with both PDGF-BB and VEGF- $\mathrm{A}_{165}$, and could be applied to functionalize glass coverslips, polyacrylamide gels, and collagen scaffolds. Moreover, each GF could be selectively activated by different types of cells, e.g., by smooth muscle cells but not fibroblasts. ${ }^{[162]}$

In all these cases, however, results were obtained by the delivery and controlled release of exogenous GFs, by tuning the binding affinity of specific aptamers. Their application for specific capture of GFs from complex mixtures and presentation to cell receptors was not tested in these studies. Conversely, Enam et al. followed this approach in order to promote wound healing via immunomodulation. ${ }^{[166]}$ In this work, PEGDA hydrogels were functionalized with antifractalkine (CX3CL1) aptamers, with the goal of sequestering and localizing this chemokine. This would, in turn, enrich the wound microenvironment, thereby specifically recruiting anti-inflammatory immune cells to the injury site. In vitro, modified gels could retain and constantly release fractalkine for at least 1 week, while in vivo, without addition of exogenous chemokine, they significantly increased the number of $\mathrm{Ly} 6 \mathrm{C}^{\mathrm{lo}} \mathrm{CX} 3 \mathrm{CR} 1^{\mathrm{hi}}$ 
nonclassical monocytes and $\mathrm{CD}^{2} 06^{+}$M2-like macrophages recruited to the injection site. This study demonstrates that the concept of modulating cell responses by locally enriching the concentration of a given factor is plausible, without delivery of any exogenous proteins or cells. ${ }^{[166]}$

Aptamers can also be useful for manipulating cell adhesion in dynamic culture systems, for example, to generate cell sheets. ${ }^{[178]}$ Reversible intermolecular hybridization is particularly important for this purpose, ensuring a controllable way to activate and inactivate adhesion motifs without any harsh conditions that could affect cellular viability. Although not using aptamers, a study has demonstrated this concept by creating DNA-antibody chimeras. In these molecules, the antibody portion was responsible for cell recognition and binding, while the oligonucleotide portion bound to another oligonucleotide sequence on functionalized culture substrates. Cell attachment to hydrogels treated with the chimeras plateaued after half an hour of incubation. Then, cell detachment could be quickly mediated by the addition of complementary oligonucleotides, which bound the chimeras more strongly than the sequences present on the hydrogel surface. ${ }^{[178]}$

Similar principles were then explored but exclusively using aptamers as affinity units. ${ }^{[179]}$ The produced sequence could switch between two conformational states depending on the addition of two different oligonucleotides. The developed aptamer, however, remained constantly bound to the supporting hydrogel, with the advantage of not being spent along the cycles of use, which occurs with other sensitive linker systems. Another advantage is that the adhesion motif detaches itself from cell receptors, instead of remaining bound as cells are released from the surface, thereby no longer affecting signaling pathways. As such, cell adhesion could be promoted by addition of the activator sequence, which partially hybridized with the hydrogel-bound aptamer. This interaction switched it "on" (activated state) and allowed recognition of $\mathrm{T}$ lymphoblastoid cells through its specific interaction with the protein tyrosine kinase receptor 7. Addition of a "recovery" sequence neutralized the activator sequence and switched the aptamer back to an inactive state, thus releasing the adhered cells in a reversible manner. ${ }^{[179]}$

In an interesting approach, $\mathrm{Li}$ et al. have recently proposed an aptamer-based dynamic cell culture system that responds to changes in the microenvironment triggered by the cells themselves, without needing the addition of exogenous stimuli. This system makes use of an aptamer sequence produced to bind adenosine triphosphate (ATP), which can be secreted by cells to participate in cellular communication and functional regulation. A complementary sequence coupled with an RGD peptide was designed to hybridize with this aptamer, allowing cell adhesion to the supporting substrate. When platelets present in the culture medium were stimulated with thrombin, they released their granule content, including ATP. This ATP then bound the aptamer, causing the release of the RGD-containing sequence and leading to a fast detachment of adhered cells (reaching $57 \%$ within $20 \mathrm{~min}) \cdot{ }^{[180]}$

The versatility of aptamers has also been explored beyond biomaterial functionalization to design functional mimetics of GFs, i.e., as agonists to GF receptors. This strategy implies the replacement of expensive and labile proteins with better alternatives that activate the same pathways and cell responses. ${ }^{[181-183]}$ Some examples of these mimetic aptamers that have been developed so far include: a divalent agonist of VEGF receptor-2, which could selectively bind, dimerize, and activate this receptor; subsequent activation of downstream Akt pathway led to upregulation of endothelial nitric oxide synthase and formation of endothelial cell capillary tubes; ${ }^{[181]}$ an HGF mimetic which, as a dimer, could induce Met receptor activation at nanomolar potency, reproducing HGF-induced cell responses such as migration and proliferation; ${ }^{[182]}$ and a 76-mer single-stranded DNA sequence that could support the selfrenewal and pluripotency characteristics of induced pluripotent stem cells, replacing FGF-2 in culture conditions. ${ }^{[183]}$

As has been shown in recent years, aptamers have increasingly appeared as valid options to develop biomaterials with not only increased affinity for GFs in general, but also specificity and dynamic programmability. These properties allow an evergreater degree of control over biomaterial biofunctionalization and, therefore, a greater precision in eliciting determined cellular responses. Despite this, some challenges still remain in their path to clinical translation. Two of the major problems pointed out so far are the lack of associated safety data, a central concern of regulatory authorities for approval of new therapies; ${ }^{[184]}$ and the lack of a clear roadmap toward large-scale good manufacturing practices grade synthesis and purification, which are required for clinical trials. ${ }^{[185]}$ However, with the approval of Macugen by the FDA in 2004, as well as several aptamers having reached phase II clinical trials, this situation might be reaching a turning point. ${ }^{[171]}$ Some inherent physicochemical characteristics of aptamers have also been mentioned as limitations that should be kept in mind. Namely rapid clearance and degradation, together with sensitivity to microenvironments, which might alter their functionality. ${ }^{[168]}$ Lastly, both aptamers and peptides are discovered through a cyclical process of affinity purification from randomly generated libraries. Depending on the number of cycles necessary to achieve adequate affinity, and with the ever-growing sizes of these libraries, a substantial amount of time and reagents could be spent on a "blind search," increasing the overall cost associated with the research. ${ }^{[186]}$

\subsubsection{Next-Generation Synthetic Platforms with Abiotic Affinity}

All the discussed methods for recognition and affinity immobilization of GFs in the previous subsections have in common been based on biological molecules as binding units, be they glycans, proteins, peptides, or oligonucleotides. Recognizing the strengths and weaknesses of the different approaches, their current limitations and stage of development have not allowed a successful clinical translation of these types of biomaterials. One particular aspect that can be deduced is that despite the increased specificity of units such as antibodies or aptamers, their low shelf-life and high cost are drawbacks. Therefore, in spite of the progressive optimization made, several research groups have started to turn to other innovative alternatives that could circumvent these issues. In this context, the possibility of precise engineering with greater control offered by synthetic polymers and advanced processing techniques have made these options attractive. ${ }^{[187,188]}$ 

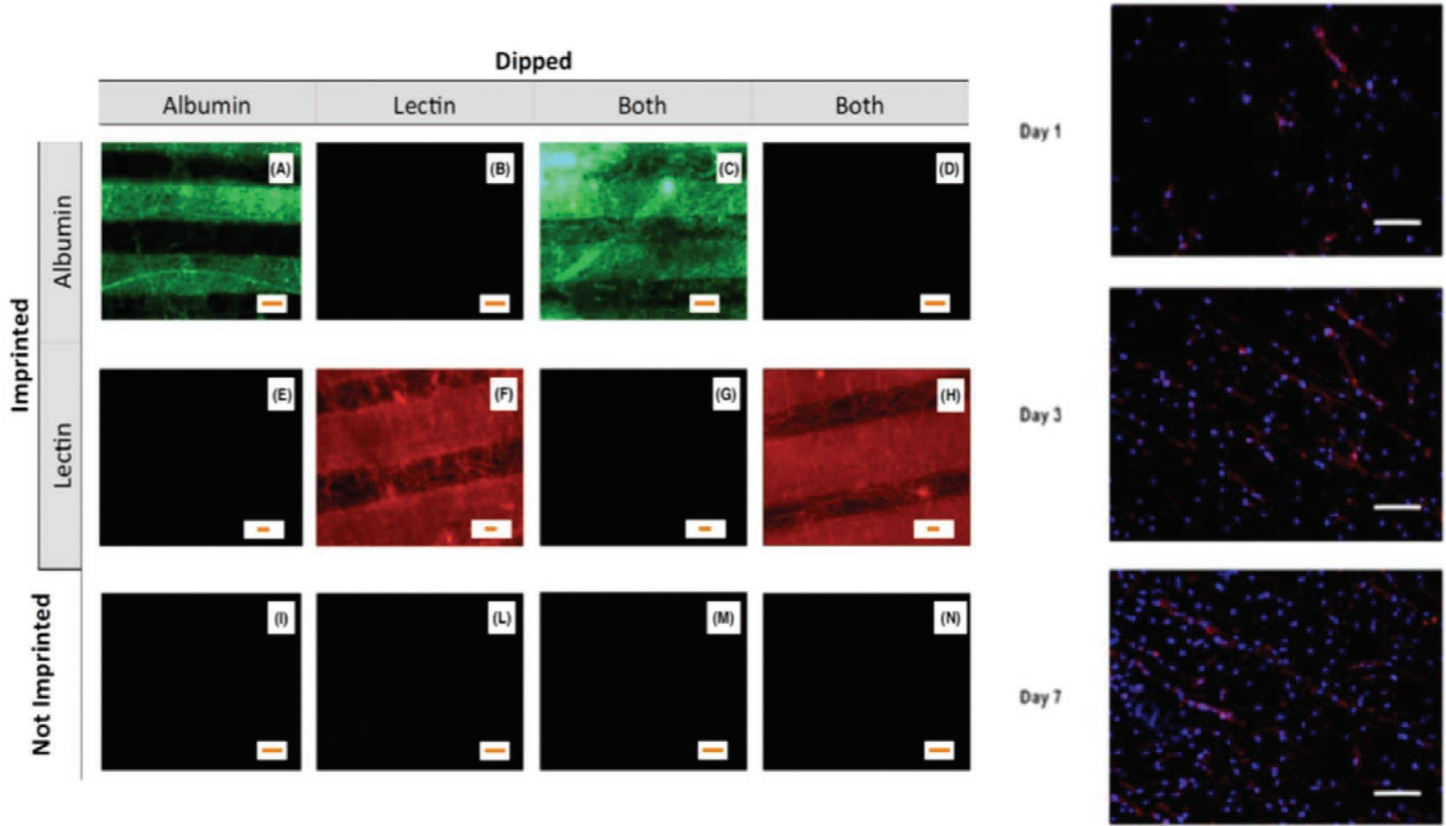

Figure 5. SMIES produced for albumin and/or lectin can successfully capture the imprinted protein from solution (left). FGF-2-imprinted SMIES increase proliferation and alignment of cultured MSCs (right). Adapted with permission. ${ }^{[178]}$ Copyright 2018, IOP Publishing.

In particular, the use of molecularly imprinted (MI) polymers (MIPs) has begun to attract some attention in the field. In the past few years, a small number of studies have shown that MIPs have the potential to create cost-effective, scalable, selective, and stable platforms for targeting specific GFs. ${ }^{[89-191]}$ MIPs are essentially synthetic receptors for a targeted molecule, being a man-made analog of natural molecules like antibodies or enzymes. They are produced by polymerizing a monomer solution in the presence of the target molecule, which is used as a template. After its extraction, the resulting polymer is endowed with complementary cavities that can selectively rebind the imprinted molecule. Although MI technology is not exactly new, it has undergone significant improvements in recent years, and has increasingly found uses in the biomedical field, particularly in biosensing applications (which constitute the bulk of published works on the matter). ${ }^{[192,193]}$ Other purposes where nanoparticles imprinted against proteins have also been successfully studied are targeted drug delivery, ${ }^{[191]}$ in vivo imaging, ${ }^{[190]}$ or molecular purification. ${ }^{[194]}$

Some recent works have introduced the advantages of MI to the modulation of cell-biomaterial interfaces via molecular recognition. Criscenti et al. combined electrospinning and softmolecular imprinting techniques to produce soft-molecularly imprinted electrospun bioactive scaffolds (SMIES) to apply in tissue regeneration. ${ }^{[189]}$ This new technology was tested with different GFs and fabricated with various polyesters, demonstrating its versatility. Briefly, a polydimethylsiloxane mold was used in order to precisely establish determined patterns on the final scaffold. This was functionalized with the target protein and used as the target for the electrospinning procedure, resulting in a patterned, imprinted fiber mesh. The process thus takes advantage of the combined techniques to generate ECM-mimetic scaffolds. SMIES were successfully imprinted with one of three GFs and tested for particular responses in different cell types. FGF-2-imprinted scaffolds significantly increased proliferation of MSCs, while BMP- 2 and TGF- $\beta 3$ were evaluated on reporter cell lines, which showed amplified luciferase activity. Both results demonstrated the differential effects of the imprinting procedure on cell behavior (Figure 5). ${ }^{[189]}$

Another good example of the use of MI was presented by Pan et al. In this work, epitope imprinting was applied to create a biointerface with dynamic bioactivity, allowing easy harvesting of cell sheets without interfering with cell viability. ${ }^{[195]}$ The authors designed a carboxyl-rich peptide (DDDGGDDD) which was used to imprint a benzamidine-based polymeric surface. This could then be used to capture another peptide, DDDGGDDDSSSSSRGDS, comprising three components. First, the previous epitope at the N-terminus ensured its interaction with the polymer. Second, a hydrophilic spacer (SSSSS) allowed its extension in culture medium and reduced unspecific adsorption. Third, an RGD sequence allowed for interaction with cell integrins. Functionalized surfaces could be used to successfully culture 3T3 fibroblasts, while nonimprinted or nonfunctionalized surfaces did not allow cell culture due to their hydrophilicity. Furthermore, addition of the epitope peptide to the culture medium allowed a dynamic ligand exchange, where it replaced the RGD-containing peptide over time. This caused the release of attached fibroblasts, with over $90 \%$ of cells detaching after $12 \mathrm{~h}$. Importantly, detached cells could then be cultured again on a new Petri dish, thus implying that the molecule-exchange-induced cell release occurs in a noninvasive manner. ${ }^{[195]}$

While they may be few, these constitute promising first steps to take advantage of synthetic materials and technologies to produce biomaterials with selective recognition properties. Many of the pitfalls that have been pointed out for other platforms 
can be bypassed by using abiotic affinity technologies, especially considering accessibility and affordability of production. ${ }^{[1]}$ Moreover, the use of one does not exclude the other; combinations of technologies such as MIPs and aptamers are already under research as well. ${ }^{[196]}$ If the low cost of MIPs can be maintained when combined with biomolecules with well-established molecular recognition properties, they may yet achieve new breakthroughs. We therefore envision a growing importance of MI in the biomedical field in the coming years, as biomaterial systems become increasingly complex and precise, and new approaches need to be sought where traditional concepts can no longer solve arising difficulties.

\section{Conclusions and Outlook}

GFs have for a long time been identified as important regulators of cell responses, in particular during processes of development, contributing to stem cell survival, proliferation, and differentiation. But attempts to use them in clinic met with largely disappointing results. The emergence of tissue engineering and biomaterials has given a new life to these promising agents, by finding ways to protect, stabilize, and increase their bioavailability. As we have explored over the course of this review, emerging trends increasingly focus on the development of platforms inspired by ECM dynamics. These strategies can more closely mimic the ways in which microenvironments modulate GF activity, shortening the distance between artificial constructs and native tissues. Herein, the main strategies being researched were described and illustrated by the most relevant and recent examples, along with associated advantages and limitations.

The use of easily accessible natural components such as heparin and fibronectin constitutes a highly versatile approach to increase effectiveness and, therefore, reduce the required doses of administered GFs. However, their promiscuity does not allow a precise steering of cell fate via biological stimulation. Instead, selective molecules designed for recognition of discrete factors, chiefly peptides and aptamers, can be leveraged in this field. The latest improvements in these technologies have allowed the paradigm to further advance toward the engineering of dynamic and biomimetic cellular niches. These include developing implants (biomaterials or engineered tissues) which can rewire endogenous regenerative mechanisms, decreasing costs while increasing biocompatibility and, ideally, therapeutic success. Another potential idea that could be explored in the future is that of "biomolecular factories," such as engineered bacteria. Their introduction in biomedical implants can provide a controllable source of desirable GFs, avoiding supplementation of exogenous proteins. Finally, we posited how new synthetic technologies, particularly MI, can be introduced into the field to circumvent existing limitations. These new additions are pushing beyond current boundaries and can possibly be one of the keys to open a new era of affordable and mass producible, yet intelligent and highly personalized constructs. It is our belief that solving this apparent paradox is indeed the crucial goal of the years to come, which could firmly establish tissue engineering as a widely accessible go-to clinical solution for people worldwide.
Moreover, the growing interest in combining perfusable microfluidic technologies with tissue engineering to develop robust in vitro models for the study of tissue and organ physiology in health and disease-the concept of organ on chipis also pushing the boundaries of biosensing technologies, allowing online monitoring. ${ }^{[197]}$ Considering that the current main area of exploration for these materials with abiotic affinity is signal transduction in biosensors, ${ }^{[193]}$ it can be anticipated that MIPs will also be particularly useful in organ-on-chip development to maintain the low cost of the sensing applications, and in cases where analytes do not have a known binding partner. ${ }^{[198]}$

\section{Acknowledgements}

The authors acknowledge the financial support from project NORTE-010145-FEDER-000021 supported by Norte Portugal Regional Operational Programme (NORTE 2020), under the PORTUGAL 2020 Partnership Agreement, through the European Regional Development Fund (ERDF); the European Union Framework Programme for Research and Innovation HORIZON 2020, under the TEAMING Grant Agreement No 739572The Discoveries CTR EU, Twinning Grant agreement No 810850Achilles, European Research Council Grant Agreement No 772817; FCT/MCTES (Fundação para a Ciência e a Tecnologia/ Ministério da Ciência, Tecnologia, e Ensino Superior), and the Fundo Social Europeu através do Programa Operacional do Capital Humano (FSE/POCH) in the framework of Ph.D. Grant PD/169/2013-PD/BD/143039/2018 for S.P.B.T., and project Grant PTDC/NAN-MAT/30595/2017.

\section{Conflict of Interest}

The authors declare no conflict of interest.

\section{Keywords}

affinity binding, biomaterials, growth factors, molecular recognition, stem cells

Received: October 30, 2019

Revised: December 20, 2019

Published online:

[1] J. R. Clegg, M. E. Wechsler, N. A. Peppas, Regener. Eng. Transl. Med. 2017, 3, 166.

[2] K. Unsicker, K. Krieglstein, Cell Signaling and Growth Factors in Development, Wiley, Weinheim, Germany 2005.

[3] S. Barrientos, O. Stojadinovic, M. S. Golinko, H. Brem, M. Tomic-Canic, Wound Repair Regener. 2008, 16, 585.

[4] M. E. Önger, B. Delibaş, A. P. Türkmen, E. Erener, B. Z. Altunkaynak, S. Kaplan, Drug Discoveries Ther. 2016, 10, 285.

[5] Z. Wang, Z. Wang, W. W. Lu, W. Zhen, D. Yang, S. Peng, NPG Asia Mater. 2017, 9, e435.

[6] NIH-U.S. National Library of Medicine, ClinicalTrials.gov (accessed: August 2019).

[7] Palifermin (marketed as Kepivance), U.S. Food Drug Adm., https://www.fda.gov/drugs/postmarket-drug-safety-informationpatients-and-providers/palifermin-marketed-kepivance (accessed: December 2019).

[8] Q. Hui, Z. Jin, X. Li, C. Liu, X. Wang, Int. J. Mol. Sci. 2018, 19, 1875. 
[9] H. Le Tuyet, T. T. Nguyen Quynh, H. Vo Hoang Minh, D. N. Th Bich, T. Do Dinh, D. Le Tan, H. L. Van, T. Le Huy, H. Doan Huu, T. N. Tran Trong, Int. Wound J. 2009, 6, 159.

[10] H. Qi, L. Yang, X. Li, X. Sun, J. Zhao, X. Hou, Z. Li, X. Yuan, Z. Cui, X. Yang, Biomater. Sci. 2019, 7, 1675.

[11] A. C. Mitchell, P. S. Briquez, J. A. Hubbell, J. R. Cochran, Acta Biomater. 2016, 30, 1.

[12] A. W. James, G. LaChaud, J. Shen, G. Asatrian, V. Nguyen, X. Zhang, K. Ting, C. Soo, Tissue Eng., Part B 2016, 22, 284.

[13] B. A. Baldo, Drug Saf. 2014, 37, 921.

[14] Public Statement-Osigraft, European Medicines Agency, https:// www.ema.europa.eu/en/medicines/human/EPAR/osigraft (accessed: December 2019).

[15] A. W. Xie, W. L. Murphy, Curr. Opin. Biomed. Eng. 2019, 10, 1.

[16] E. Witsch, M. Sela, Y. Yarden, Physiology 2010, 25, 85.

[17] S. J. Allen, D. Dawbarn, Clin. Sci. 2006, 110, 175.

[18] B. Lu, P. T. Pang, N. H. Woo, Nat. Rev. Neurosci. 2005, 6, 603.

[19] M. Horiguchi, M. Ota, D. B. Rifkin, J. Biochem. 2012, 152, 321.

[20] M. H. Ross, W. Pawlina, Histology: A Text and Atlas: With Correlated Cell and Molecular Biology, 7th ed., Lippincott Wiliams \& Wilkins, Baltimore, MD 2006.

[21] H. Wolfenson, B. Yang, M. P. Sheetz, Annu. Rev. Physiol. 2019, 81, 585.

[22] M. J. Dalby, A. J. García, M. Salmeron-Sanchez, Nat. Rev. Mater. 2018, 3, 17091.

[23] J. Zhu, R. A. F. Clark, J. Invest. Dermatol. 2014, 134, 895.

[24] U. Lindahl, J. Couchman, K. Kimata, J. D. Esko, in Essentials of Glycobiology (Eds: A. Varki, R. D. Cummings, D. J. Esko, P. Stanley, G. Hart, M. Aebi, A. G. Darvill, T. Kinoshita, N. H. Packer, J. H. Prestegard, R. L. Schnaar, P. H. Seeberger) CSHL Press, New York, NY 2017.

[25] D. G. Belair, N. N. Le, W. L. Murphy, Chem. Commun. 2014, 50, 15651.

[26] M. Hajimiri, S. Shahverdi, G. Kamalinia, R. Dinarvand, J. Biomed. Mater. Res., Part A 2015, 103, 819.

[27] A. Cipitria, M. Salmeron-Sanchez, Adv. Healthcare Mater. 2017, 6, 1700052.

[28] T. G. Kapp, F. Rechenmacher, S. Neubauer, O. V. Maltsev, E. A. Cavalcanti-Adam, R. Zarka, U. Reuning, J. Notni, H.-J. Wester, C. Mas-Moruno, J. Spatz, B. Geiger, H. Kessler, Sci. Rep. 2017, 7, 39805.

[29] J. Ivaska, J. Heino, Annu. Rev. Cell Dev. Biol. 2011, 27, 291.

[30] H. Wang, H. Jin, A. C. Rapraeger, J. Biol. Chem. 2015, 290, 26103.

[31] L. Moro, M. Venturino, C. Bozzo, L. Silengo, F. Altruda, L. Beguinot, G. Tarone, P. Defilippi, EMBO J. 1998, 17, 6622.

[32] G. A. Hudalla, W. L. Murphy, Adv. Funct. Mater. 2011, 21, 1754.

[33] M. E. Wechsler, J. R. Clegg, N. A. Peppas, in Encyclopedia of Tissue Engineering and Regenerative Medicine, Academic Press, London 2019, pp. 77-86.

[34] K. Lee, E. A. Silva, D. J. Mooney, J. R. Soc., Interface 2011, 8, 153.

[35] P. A. Turner, J. S. Thiele, J. P. Stegemann, J. Biomater. Sci., Polym. Ed. 2017, 28, 1826

[36] W. J. King, P. H. Krebsbach, Adv. Drug Delivery Rev. 2012, 64, 1239.

[37] T.-M. De Witte, L. E. Fratila-Apachitei, A. A. Zadpoor, N. A. Peppas, Regener. Biomater. 2018, 5, 197.

[38] J. Aragón, S. Salerno, L. De Bartolo, S. Irusta, G. Mendoza, J. Colloid Interface Sci. 2018, 531, 126.

[39] X. Feng, J. Li, X. Zhang, T. Liu, J. Ding, X. Chen, J. Controlled Release 2019, 302, 19.

[40] W. L. Murphy, M. C. Peters, D. H. Kohn, D. J. Mooney, Biomaterials 2000, 21, 2521.

[41] J. W. Choi, Y. S. Kim, J. K. Park, E. H. Song, J. H. Park, M. S. Kim, Y. S. Shin, C.-H. Kim, Macromol. Biosci. 2017, 17, 1600163.

[42] F. Asghari, M. Samiei, K. Adibkia, A. Akbarzadeh, S. Davaran, Artif. Cells, Nanomed., Biotechnol. 2017, 45, 185.

[43] D. Qu, J. P. Zhu, H. R. Childs, H. H. Lu, Acta Biomater. 2019, 93, 111.
[44] E. Carenza, O. Jordan, P. Martínez-San Segundo, R. Jiř́k, Z. Starčuk jr., G. Borchard, A. Rosell, A. Roig, J. Mater. Chem. B 2015, 3, 2538

[45] S. J. Lee, D. Lee, T. R. Yoon, H. K. Kim, H. H. Jo, J. S. Park, J. H. Lee, W. D. Kim, I. K. Kwon, S. A. Park, Acta Biomater. 2016 40, 182.

[46] B.-B. Seo, J.-T. Koh, S.-C. Song, Biomaterials 2017, 122, 91.

[47] O. P. Oommen, S. Wang, M. Kisiel, M. Sloff, J. Hilborn, O. P. Varghese, Adv. Funct. Mater. 2013, 23, 1273.

[48] H. J. Yan, T. Casalini, G. Hulsart-Billström, S. Wang, O. P. Oommen, M. Salvalaglio, S. Larsson, J. Hilborn, O. P. Varghese, Biomaterials 2018, 161, 190.

[49] J. H. Lee, J.-H. Park, M. Eltohamy, R. Perez, E.-J. Lee, H.-W. Kim, RSC Adv. 2013, 3, 24202.

[50] D. Liu, M. Nikoo, G. Boran, P. Zhou, J. M. Regenstein, Annu. Rev. Food Sci. Technol. 2015, 6, 527.

[51] T. Kobayashi, M. Mizuta, N. Hiwatashi, Y. Kishimoto, T. Nakamura, S. Kanemaru, S. Hirano, Auris, Nasus, Larynx 2017, 44, 86.

[52] R. T. Annamalai, P. A. Turner, W. F. Carson, B. Levi, S. Kunkel, J. P. Stegemann, Biomaterials 2018, 161, 216.

[53] S. Gnavi, L. di Blasio, C. Tonda-Turo, A. Mancardi, L. Primo, G. Ciardelli, G. Gambarotta, S. Geuna, I. Perroteau, J. Tissue Eng. Regener. Med. 2017, 11, 459

[54] J. P. Yoon, C. H. Lee, J. W. Jung, H. J. Lee, Y. S. Lee, J. Y. Kim, G. Y. Park, J. H. Choi, S. W. Chung, Am. J. Sports Med. 2018, 46, 1441.

[55] D. Cizkova, L. Slovinska, I. Grulova, M. Salzet, S. Cikos, O. Kryukov, S. Cohen, J. Tissue Eng. Regener. Med. 2015, 9, 918.

[56] S. Felder, H. Masasa, A. Orenbuch, N. Levaot, M. Shachar Goldenberg, S. Cohen, Biomaterials 2019, 205, 11

[57] Z. Wang, C. Li, J. Xu, K. Wang, X. Lu, H. Zhang, S. Qu, G. Zhen, F. Ren, Chem. Mater. 2015, 27, 848.

[58] Z. Ding, Z. Fan, X. Huang, Q. Lu, W. Xu, D. L. Kaplan, ACS Appl. Mater. Interfaces 2016, 8, 24463.

[59] J. Sun, H. Tan, Materials 2013, 6, 1285.

[60] P. R. Sivashankari, M. Prabaharan, Int. J. Biol. Macromol. 2016, 93, 1382.

[61] J. O. Buitrago, K. D. Patel, A. El-Fiqi, J.-H. Lee, B. Kundu, H.-H. Lee, H.-W. Kim, Acta Biomater. 2018, 69, 218.

[62] H. Tian, J. Du, J. Wen, Y. Liu, S. R. Montgomery, T. P. Scott, B. Aghdasi, C. Xiong, A. Suzuki, T. Hayashi, M. Ruangchainikom, K. Phan, G. Weintraub, A. Raed, S. S. Murray, M. D. Daubs, X. Yang, X. B. Yuan, J. C. Wang, Y. Lu, ACS Nano 2016, 10, 7362.

[63] M. Yadlapati, C. Biguetti, F. Cavalla, F. Nieves, C. Bessey, P. Bohluli, G. P. Garlet, A. Letra, W. D. Fakhouri, R. M. Silva, J. Endod. 2017, 43, 77.

[64] C. Monge, J. Almodóvar, T. Boudou, C. Picart, Adv. Healthcare Mater. 2015, 4, 811.

[65] J. Zeng, M. Matsusaki, Polym. Chem. 2019, 10, 2960.

[66] S. Zhao, F. Caruso, L. Dähne, G. Decher, B. G. De Geest, J. Fan, N. Feliu, Y. Gogotsi, P. T. Hammond, M. C. Hersam, A. Khademhosseini, N. Kotov, S. Leporatti, Y. Li, F. Lisdat, L. M. Liz-Marzán, S. Moya, P. Mulvaney, A. L. Rogach, S. Roy, D. G. Shchukin, A. G. Skirtach, M. M. Stevens, G. B. Sukhorukov, P. S. Weiss, Z. Yue, D. Zhu, W. J. Parak, ACS Nano 2019, 13, 6151.

[67] G. Cheng, C. Yin, H. Tu, S. Jiang, Q. Wang, X. Zhou, X. Xing, C. Xie, X. Shi, Y. Du, H. Deng, Z. Li, ACS Nano 2019, 13, 6372.

[68] M. Bouyer, R. Guillot, J. Lavaud, C. Plettinx, C. Olivier, V. Curry, J. Boutonnat, J.-L. Coll, F. Peyrin, V. Josserand, G. Bettega, C. Picart, Biomaterials 2016, 104, 168.

[69] J. Min, K. Y. Choi, E. C. Dreaden, R. F. Padera, R. D. Braatz, M. Spector, P. T. Hammond, ACS Nano 2016, 10, 4441.

[70] Z. Yuan, Y. H. Tsou, X. Q. Zhang, S. Huang, Y. Yang, M. Gao, W. Ho, Q. Zhao, X. Ye, X. Xu, ACS Appl. Mater. Interfaces 2019, 11, 38429. 
[71] T. R. Ham, M. Farrag, N. D. Leipzig, Acta Biomater. 2017, 53, 140.

[72] B. Choi, S. Kim, J. Fan, T. Kowalski, F. Petrigliano, D. Evseenko, M. Lee, Biomater. Sci. 2015, 3, 742.

[73] K. S. Masters, Macromol. Biosci. 2011, 11, 1149.

[74] B. P. Mahadik, S. Pedron Haba, L. J. Skertich, B. A. C. Harley, Biomaterials 2015, 67, 297.

[75] B. V. Sridhar, N. R. Doyle, M. A. Randolph, K. S. Anseth, J. Biomed. Mater. Res., Part A 2014, 102, 4464

[76] B. E. B. Jensen, K. Edlund, A. N. Zelikin, Biomaterials 2015, 49, 113.

[77] C. S. Kim, I. P. Mitchell, A. W. Desotell, P. K. Kreeger, K. S. Masters, FASEB J. 2016, 30, 2580.

[78] H. J. Lee, G. M. Fernandes-Cunha, I. Putra, W. G. Koh, D. Myung, ACS Appl. Mater. Interfaces 2017, 9, 23389.

[79] M. Wöltje, M. Böbel, M. Bienert, S. Neuss, D. Aibibu, C. Cherif, J. Biomed. Mater. Res., Part A 2018, 106, 2643.

[80] A. M. McCormick, N. A. Jarmusik, N. D. Leipzig, Acta Biomater. 2015, 28, 33.

[81] S. Noel, C. Fortier, F. Murschel, A. Belzil, G. Gaudet, M. Jolicoeur, G. De Crescenzo, Acta Biomater. 2016, 37, 69.

[82] A. R. Armiento, M. J. Stoddart, M. Alini, D. Eglin, Acta Biomater. 2018, 65, 1.

[83] J. D. Weaver, D. M. Headen, J. Aquart, C. T. Johnson, L. D. Shea, H. Shirwan, A. J. García, Sci. Adv. 2017, 3, el 700184.

[84] M. M. Adil, T. Vazin, B. Ananthanarayanan, G. M. C. Rodrigues, A. T. Rao, R. U. Kulkarni, E. W. Miller, S. Kumar, D. V. Schaffer, Biomaterials 2017, 136, 1.

[85] J. C. Grim, T. E. Brown, B. A. Aguado, D. A. Chapnick, A. L. Viert, X. Liu, K. S. Anseth, ACS Cent. Sci. 2018, 4, 909.

[86] R. Mittermayr, P. Slezak, N. Haffner, D. Smolen, J. Hartinger, A. Hofmann, J. Schense, D. Spazierer, J. Gampfer, A. Goppelt, H. Redl, Acta Biomater. 2016, $29,11$.

[87] H. G. Schmoekel, F. E. Weber, J. C. Schense, K. W. Grätz, P. Schawalder, J. A. Hubbell, Biotechnol. Bioeng. 2005, 89, 253.

[88] L. Karfeld-Sulzer, B. Siegenthaler, C. Ghayor, F. Weber, Materials 2015, 8, 977.

[89] M. Peeters, S. E. L. Detiger, L. S. Karfeld-Sulzer, T. H. Smit, A. Yayon, F. E. Weber, M. N. Helder, BioRes. Open Access 2015, 4, 398.

[90] J. A. Shadish, G. M. Benuska, C. A. DeForest, Nat. Mater. 2019, 18, 1005.

[91] S. V. Wegner, O. I. Sentürk, J. P. Spatz, Sci. Rep. 2016, 5, 18309.

[92] S. Palta, R. Saroa, A. Palta, Indian J. Anaesth. 2014, 58, 515.

[93] Z. A. Cheng, A. Alba-Perez, C. Gonzalez-Garcia, H. Donnelly, V. Llopis-Hernandez, V. Jayawarna, P. Childs, D. W. Shields, M. Cantini, L. Ruiz-Cantu, A. Reid, J. F. C. Windmill, E. S. Addison, S. Corr, W. G. Marshall, M. J. Dalby, M. Salmeron-Sanchez, Adv. Sci. 2018, 6, 1800361.

[94] P. S. Briquez, J. A. Hubbell, M. M. Martino, Adv. Wound Care 2015, 4, 479.

[95] M. M. Martino, S. Brkic, E. Bovo, M. Burger, D. J. Schaefer, T. Wolff, L. Gürke, P. S. Briquez, H. M. Larsson, R. Gianni-Barrera, J. A. Hubbell, A. Banfi, Front. Bioeng. Biotechnol. 2015, 3, 45.

[96] C. Silva, A. Carretero, D. Soares da Costa, R. L. Reis, R. Novoa-Carballal, I. Pashkuleva, Acta Biomater. 2017, 63, 283.

[97] S. E. Sakiyama-Elbert, Acta Biomater. 2014, 10, 1581.

[98] R. Chen, Y. Yu, W. Zhang, Y. Pan, J. Wang, Y. Xiao, C. Liu, Acta Biomater. 2018, 80, 108.

[99] F. Copes, P. Chevallier, C. Loy, D. Pezzoli, F. Boccafoschi, D. Mantovani, Front. Bioeng. Biotechnol. 2019, 7, 74.

[100] J. R. Krieger, M. E. Ogle, J. McFaline-Figueroa, C. E. Segar, J. S. Temenoff, E. A. Botchwey, Biomaterials 2016, 77, 280.

[101] A. K. Jha, K. M. Tharp, J. Ye, J. L. Santiago-Ortiz, W. M. Jackson, A. Stahl, D. V. Schaffer, Y. Yeghiazarians, K. E. Healy, Biomaterials 2015, 47, 1.

[102] A. K. Jha, A. Mathur, F. L. Svedlund, J. Ye, Y. Yeghiazarians, K. E. Healy, J. Controlled Release 2015, 209, 308.

[103] S. Browne, A. K. Jha, K. Ameri, S. G. Marcus, Y. Yeghiazarians, K. E. Healy, PLoS One 2018, 13, e0194679.
[104] Y. Feng, Q. Li, D. Wu, Y. Niu, C. Yang, L. Dong, C. Wang, Biomaterials 2017, 134, 128.

[105] B. Newland, P. B. Welzel, H. Newland, C. Renneberg, P. Kolar, M. Tsurkan, A. Rosser, U. Freudenberg, C. Werner, Small 2015, 11, 5047.

[106] S. Font Tellado, S. Chiera, W. Bonani, P. S. P. Poh, C. Migliaresi, A. Motta, E. R. Balmayor, M. van Griensven, Acta Biomater. 2018, $72,150$.

[107] J. C. Bragg, H. Kweon, Y. Jo, K. G. Lee, C.-C. Lin, RSC Adv. 2016, 6, 114353.

[108] Y. Arisaka, J. Kobayashi, K. Ohashi, K. Tatsumi, K. Kim, Y. Akiyama, M. Yamato, T. Okano, Regen. Ther. 2016, 3, 97.

[109] Y. Hwang, M. C. Goh, M. Kim, G. Tae, Biomaterials 2018, 165, 94.

[110] Y. Liu, H.-P. Sun, J.-Y. Xu, Y.-M. Li, X. Xie, F.-L. Deng, L.-Z. Deng, L.-M. Zhang, Int. J. Nanomed. 2016, 11, 1147.

[111] A. N. Rindone, B. Kachniarz, C. C. Achebe, R. C. Riddle, A. N. O'Sullivan, A. H. Dorafshar, W. L. Grayson, Adv. Healthcare Mater. 2019, 8, 1801565.

[112] B. Mulloy, J. Hogwood, E. Gray, R. Lever, C. P. Page, Pharmacol. Rev. 2016, 68, 76.

[113] S. Mohamed, D. Coombe, Pharmaceuticals 2017, 10, 78.

[114] S. S. Lee, T. Fyrner, F. Chen, Z. Álvarez, E. Sleep, D. S. Chun, J. A. Weiner, R. W. Cook, R. D. Freshman, M. S. Schallmo, K. M. Katchko, A. D. Schneider, J. T. Smith, C. Yun, G. Singh, S. Z. Hashmi, M. T. McClendon, Z. Yu, S. R. Stock, W. K. Hsu, E. L. Hsu, S. I. Stupp, Nat. Nanotechnol. 2017, 12, 821.

[115] S. Kim, Z.-K. Cui, P. J. Kim, L. Y. Jung, M. Lee, Acta Biomater. 2018, $72,45$.

[116] N. Lohmann, E. Wandel, R. A. Ferrer, J. C. Simon, S. Franz, L. Schirmer, P. Atallah, C. Werner, U. Freudenberg, Sci. Transl. Med. 2017, 9, eaai9044.

[117] G. A. McCanney, M. A. McGrath, T. D. Otto, R. Burchmore, E. A. Yates, C. D. Bavington, H. J. Willison, J. E. Turnbull, S. C. Barnett, Glia 2019, 67, 668.

[118] T. E. Rinker, B. D. Philbrick, M. H. Hettiaratchi, D. M. Smalley, T. C. McDevitt, J. S. Temenoff, Acta Biomater. 2018, 68, 125.

[119] M. Al-Jarsha, V. Moulisová, A. Leal-Egaña, A. Connell, K. B. Naudi, A. F. Ayoub, M. J. Dalby, M. Salmerón-Sánchez, ACS Biomater. Sci. Eng. 2018, 4, 1812.

[120] D. L. Mooradian, R. C. Lucas, J. A. Weatherbee, L. T. Furcht, J. Cell. Biochem. 1989, 41, 189.

[121] S. L. Dallas, P. Sivakumar, C. J. P. Jones, Q. Chen, D. M. Peters, D. F. Mosher, M. J. Humphries, C. M. Kielty, J. Biol. Chem. 2005, 280, 18871.

[122] E. S. Wijelath, S. Rahman, M. Namekata, J. Murray, T. Nishimura, Z. Mostafavi-Pour, Y. Patel, Y. Suda, M. J. Humphries, M. Sobel, Circ. Res. 2006, 99, 853 .

[123] E. S. Wijelath, J. Murray, S. Rahman, Y. Patel, A. Ishida, K. Strand, S. Aziz, C. Cardona, W. P. Hammond, G. F. Savidge, S. Rafii, M. Sobel, Circ. Res. 2002, 91, 25.

[124] M. Mitsi, Z. Hong, C. E. Costello, M. A. Nugent, Biochemistry 2006, 45, 10319

[125] M. M. Martino, J. A. Hubbell, FASEB J. 2010, 24, 4711.

[126] M. M. Martino, P. S. Briquez, A. Ranga, M. P. Lutolf, J. A. Hubbell, Proc. Natl. Acad. Sci. USA 2013, 110, 4563.

[127] V. Llopis-Hernández, M. Cantini, C. González-García, Z. A. Cheng, J. Yang, P. M. Tsimbouri, A. J. García, M. J. Dalby, M. SalmerónSánchez, Sci. Adv. 2016, 2, e1600188.

[128] R. Sabater i Serra, L. León-Boigues, A. Sánchez-Laosa, L. Gómez-Estrada, J. L. Gómez Ribelles, M. Salmeron-Sanchez, G. Gallego Ferrer, Colloids Surf., B 2016, 148, 324.

[129] V. Moulisová, C. Gonzalez-García, M. Cantini, A. Rodrigo-Navarro, J. Weaver, M. Costell, R. Sabater i Serra, M. J. Dalby, A. J. García, M. Salmerón-Sánchez, Biomaterials 2017, 126, 61.

[130] S. Trujillo, C. Gonzalez-Garcia, P. Rico, A. Reid, J. Windmill, M. J. Dalby, M. Salmeron-Sanchez, bioRxiv 687244, 2019. 
[131] M. Kisiel, M. M. Martino, M. Ventura, J. A. Hubbell, J. Hilborn, D. A. Ossipov, Biomaterials 2013, 34, 704.

[132] M. M. Martino, F. Tortelli, M. Mochizuki, S. Traub, D. Ben-David, G. A. Kuhn, R. Muller, E. Livne, S. A. Eming, J. A. Hubbell, Sci. Transl. Med. 2011, 3, 100ra89.

[133] J. J. Hay, A. Rodrigo-Navarro, M. Petaroudi, A. V. Bryksin, A. J. García, T. H. Barker, M. J. Dalby, M. Salmeron-Sanchez, Adv. Mater. 2018, 30, 1804310.

[134] R. B. Dodd, T. Wilkinson, D. J. Schofield, BioDrugs 2018, 32, 339.

[135] D. A. Quinteros, J. M. Bermúdez, S. Ravetti, A. Cid, D. A. Allemandi, S. D. Palma, in Nanostructures for Drug Delivery (Eds: E. Andronescu, A. M. Grumezescu), Elsevier, Amsterdam 2017, pp. 807-833.

[136] C. Oliveira, A. R. Costa-Pinto, R. L. Reis, A. Martins, N. M. Neves, Biomacromolecules 2014, 15, 2196.

[137] M. R. Casanova, M. Alves da Silva, A. R. Costa-Pinto, R. L. Reis, A. Martins, N. M. Neves, Mater. Sci. Eng., C 2019, 98, 1169.

[138] E. Bacelo, M. A. Da Silva, C. Cunha, S. Faria, A. Carvalho, R. L. Reis, A. Martins, N. M. Neves, Nanomaterials 2019, 9, 567.

[139] S. Awwad, U. Angkawinitwong, Pharmaceutics 2018, 10, 83

[140] M. K. Alam, M. Brabant, R. S. Viswas, K. Barreto, H. Fonge, C. Ronald Geyer, BMC Biotechnol. 2018, 18, 55.

[141] A. Borek, A. Sokolowska-Wedzina, G. Chodaczek, J. Otlewski, PLoS One 2018, 13, e0192194.

[142] S. Kordi, M. Rahmati-Yamchi, M. Asghari Vostakolaei, A. Barzegari, J. Abdolalizadeh, Adv. Pharm. Bull. 2019, 9, 64

[143] S. S. Lee, E. L. Hsu, M. Mendoza, J. Ghodasra, M. S. Nickoli, A. Ashtekar, M. Polavarapu, J. Babu, R. M. Riaz, J. D. Nicolas, D. Nelson, S. Z. Hashmi, S. R. Kaltz, J. S. Earhart, B. R. Merk, J. S. Mckee, S. F. Bairstow, R. N. Shah, W. K. Hsu, S. I. Stupp Adv. Healthcare Mater. 2015, 4, 131.

[144] J. F. Crispim, S. C. Fu, Y. W. Lee, H. A. M. Fernandes, P. Jonkheijm, P. S. H. Yung, D. B. F. Saris, Am. J. Sports Med. 2018, 46, 2905.

[145] J. Crispim, H. A. M. Fernandes, S. C. Fu, Y. W. Lee, P. Jonkheijm, D. B. F. Saris, Acta Biomater. 2017, 53, 165.

[146] M. Xiao, J. Xiao, G. Wu, Y. Ke, L. Fang, C. Deng, H. Liao, Colloids Surf., B 2018, 166, 254.

[147] G. Wu, M. Xiao, J. Xiao, L. Guo, Y. Ke, H. Li, L. Fang, C. Deng, H. Liao, Mater. Sci. Eng., C 2018, 83, 67.

[148] J. Chen, Y. Li, B. Wang, J. Yang, B. C. Heng, Z. Yang, Z. Ge, J. Lin, J. Mater. Chem. B 2018, 6, 675

[149] C. Suttinont, Y. Mashimo, M. Mie, E. Kobatake, Biomed Res. Int 2015, 2015, 208089

[150] G. A. Hudalla, J. T. Koepsel, W. L. Murphy, Adv. Mater. 2011, 23, 5415

[151] J. Ishihara, A. Ishihara, K. Fukunaga, K. Sasaki, M. J. V. White, P. S. Briquez, J. A. Hubbell, Nat. Commun. 2018, 9, 2163.

[152] P. Lequoy, F. Murschel, B. Liberelle, S. Lerouge, G. De Crescenzo, Acta Biomater. 2016, 29, 239.

[153] C. Addi, F. Murschel, B. Liberelle, N. Riahi, G. De Crescenzo, Acta Biomater. 2017, 50, 198.

[154] P. S. Lienemann, Y. R. Devaud, R. Reuten, B. R. Simona, M. Karlsson, W. Weber, M. Koch, M. P. Lutolf, V. Milleret, M. Ehrbar, Integr. Biol. 2015, 7, 101.

[155] D. G. Belair, N. N. Le, W. L. Murphy, Biomater. Sci. 2016, 4, 819.

[156] N. Zhao, J. Coyne, M. Xu, X. Zhang, A. Suzuki, P. Shi, J. Lai, G. H. Fong, N. Xiong, Y. Wang, Chem. Mater. 2019, 31, 1006.

[157] N. Zhao, A. Suzuki, X. Zhang, P. Shi, L. Abune, J. Coyne, H. Jia, N. Xiong, G. Zhang, Y. Wang, ACS Appl. Mater. Interfaces 2019, 11, 18123.

[158] N. Zhao, M. R. Battig, M. Xu, X. Wang, N. Xiong, Y. Wang, Macromol. Biosci. 2017, 17, 1700201

[159] X. Zhang, M. R. Battig, N. Chen, E. R. Gaddes, K. L. Duncan, Y. Wang, Biomacromolecules 2016, 17, 778.

[160] L. Abune, N. Zhao, J. Lai, B. Peterson, S. Szczesny, Y. Wang, ACS Biomater. Sci. Eng. 2019, 5, 2382.

[161] Z. Zhang, C. Liu, C. Yang, Y. Wu, F. Yu, Y. Chen, J. Du, ACS Appl. Mater. Interfaces 2018, 10, 8546.
[162] A. Stejskalová, N. Oliva, F. J. England, B. D. Almquist, Adv. Mater. 2019, 31, 1806380.

[163] M. R. Battig, B. Soontornworajit, Y. Wang, J. Am. Chem. Soc. 2012, 134,12410

[164] M. R. Battig, Y. Huang, N. Chen, Y. Wang, Biomaterials 2014, 35, 8040.

[165] X. Wang, X. Song, T. Li, J. Chen, G. Cheng, L. Yang, C. Chen, Am. J. Sports Med. 2019, 47, 2316.

[166] S. F. Enam, J. R. Krieger, T. Saxena, B. E. Watts, C. E. Olingy, E. A. Botchwey, R. V. Bellamkonda, Biomaterials 2017, 142, 52.

[167] Y.-M. Fang, D.-Q. Lin, S.-J. Yao, J. Chromatogr. A 2018, 1571, 1.

[168] Z. Jiang, J. Guan, J. Qian, C. Zhan, Biomater. Sci. 2019, 7, 461.

[169] C.-H. Wu, I.-J. Liu, R.-M. Lu, H.-C. Wu, J. Biomed. Sci. 2016, 23, 8.

[170] J. C. Rosch, E. K. Hollmann, E. S. Lippmann, Exp. Biol. Med. 2016, 241, 962.

[171] A. Parashar, J. Clin. Diagn. Res. 2016, 10, BE01.

[172] D. Molin, M. Post, Curr. Opin. Pharmacol. 2007, 7, 158.

[173] H. Al Sabti, J. Cardiothorac. Surg. 2007, 2, 49.

[174] E. W. M. Ng, D. T. Shima, P. Calias, E. T. Cunningham, D. R. Guyer, A. P. Adamis, Nat. Rev. Drug Discovery 2006, 5, 123.

[175] Y. Li, H. Meng, Y. Liu, B. P. Lee, Sci. World J. 2015, 2015, 685690.

[176] C. H. Park, K. M. Woo, in Biomimetic Medical Materials. Advances in Experimental Medicine and Biology (Ed: I. Noh), Vol. 1064, Springer, Singapore 2018, pp. 253-261

[177] B. Hinz, Matrix Biol. 2015, 47, 54.

[178] Z. Zhang, S. Li, N. Chen, C. Yang, Y. Wang, Biomacromolecules 2013, 14, 1174

[179] S. Li, E. R. Gaddes, N. Chen, Y. Wang, Angew. Chem. 2015, 127, 6055; Angew. Chem., Int. Ed. 2015, 54, 5957.

[180] W. Li, J. Wang, J. Ren, X. Qu, Chem. Sci. 2015, 6, 6762.

[181] V. Ramaswamy, A. Monsalve, L. Sautina, M. S. Segal, J. Dobson, J. B. Allen, Nucleic Acid Ther. 2015, 25, 227.

[182] R. Ueki, A. Ueki, N. Kanda, S. Sando, Angew. Chem. 2016, 128 , 589; Angew. Chem., Int. Ed. 2016, 55, 579.

[183] R. Ueki, S. Atsuta, A. Ueki, J. Hoshiyama, J. Li, Y. Hayashi, S. Sando, Chem. Commun. 2019, 55, 2672.

[184] J. Zhou, J. Rossi, Nat. Rev. Drug Discovery 2017, 16, 181.

[185] Q. Zhang, R. Landgraf, Pharmaceuticals 2012, 5, 493.

[186] M. H. S. Segler, T. Kogej, C. Tyrchan, M. P. Waller, ACS Cent. Sci. 2018, 4, 120.

[187] P. Latza, P. Gilles, T. Schaller, T. Schrader, Chem. - Eur.J. 2014, 20, 11479.

[188] D. Tamburro, C. Fredolini, V. Espina, T. A. Douglas, A. Ranganathan, L. Ilag, W. Zhou, P. Russo, B. H. Espina, G. Muto, E. F. Petricoin, L. A. Liotta, A. Luchini, J. Am. Chem. Soc. 2011, 133, 19178

[189] G. Criscenti, C. De Maria, A. Longoni, C. A. van Blitterswijk, H. A. M. Fernandes, G. Vozzi, L. Moroni, Biofabrication 2018, 10, 045005.

[190] A. Cecchini, V. Raffa, F. Canfarotta, G. Signore, S. Piletsky, M. P. Macdonald, A. Cuschieri, Nano Lett. 2017, 17, 2307.

[191] F. Canfarotta, L. Lezina, A. Guerreiro, J. Czulak, A. Petukhov, A. Daks, K. Smolinska-Kempisty, A. Poma, S. Piletsky, N. A. Barlev, Nano Lett. 2018, 18, 4641.

[192] J. J. BelBruno, Chem. Rev. 2018, 119, 94.

[193] O. S. Ahmad, T. S. Bedwell, C. Esen, A. Garcia-Cruz, S. A. Piletsky, Trends Biotechnol. 2019, 37, 294.

[194] S. Schwark, W. Sun, J. Stute, D. Lutkemeyer, M. Ulbricht, B. Sellergren, RSC Adv. 2016, 6, 53162.

[195] G. Pan, S. Shinde, S. Y. Yeung, M. Jakštaitè, Q. Li, A. G. Wingren, B. Sellergren, Angew. Chem. 2017, 129, 16175; Angew. Chem., Int. Ed. 2017, 56, 15959.

[196] A. Poma, H. Brahmbhatt, H. M. Pendergraff, J. K. Watts, N. W. Turner, Adv. Mater. 2015, 27, 750.

[197] B. Zhang, A. Korolj, B. F. L. Lai, M. Radisic, Nat. Rev. Mater. 2018, 3, 257 .

[198] H. R. Culver, J. R. Clegg, N. A. Peppas, Acc. Chem. Res. 2017, 50, 170. 